\title{
SUVR2 is involved in transcriptional gene silencing by associating with SNF2-related chromatin-remodeling proteins in Arabidopsis
}

Yong-Feng Han ${ }^{1, *}$, Kun Dou ${ }^{1, *}$, Ze-Yang Ma ${ }^{1}$, Su-Wei Zhang ${ }^{1}$, Huan-Wei Huang ${ }^{1}$, Lin Li $^{1}$, Tao Cai ${ }^{1}$, She Chen ${ }^{1}$, Jian-Kang $\mathrm{Zhu}^{2,3}$, Xin-Jian $\mathrm{He}^{1}$

${ }^{I}$ National Institute of Biological Sciences, Beijing 102206, China; ${ }^{2}$ Department of Horticulture and Landscape Architecture, Purdue University, West Lafayette, IN 47907, USA; ${ }^{3}$ Shanghai Center for Plant Stress Biology and Institute of Plant Physiology and Ecology, Shanghai Institutes for Biological Sciences, Chinese Academy of Sciences, Shanghai 200032, China

The SU(VAR)3-9-like histone methyltransferases usually catalyze repressive histone H3K9 methylation and are involved in transcriptional gene silencing in eukaryotic organisms. We identified a putative SU(VAR)3-9-like histone methyltransferase SUVR2 by a forward genetic screen and demonstrated that it is involved in transcriptional gene silencing at genomic loci targeted by RNA-directed DNA methylation (RdDM). We found that SUVR2 has no histone methyltransferase activity and the conserved catalytic sites of SUVR2 are dispensable for the function of SUVR2 in transcriptional silencing. SUVR2 forms a complex with its close homolog SUVR1 and associate with three previously uncharacterized SNF2-related chromatin-remodeling proteins CHR19, CHR27, and CHR28. SUVR2 was previously thought to be a component in the RdDM pathway. We demonstrated that SUVR2 contributes to transcriptional gene silencing not only at a subset of RdDM target loci but also at many RdDM-independent target loci. Our study suggests that the involvement of SUVR2 in transcriptional gene silencing is related to nucleosome positioning mediated by its associated chromatin-remodeling proteins.

Keywords: SUVR1; SUVR2; CHR19; DNA methylation; chromatin remodeling; transcriptional gene silencing Cell Research (2014) 24:1445-1465. doi:10.1038/cr.2014.156; published online 25 November 2014

\section{Introduction}

DNA methylation, histone H3K9 methylation and other repressive histone marks are involved in transcriptional silencing of transposable elements (TEs) and other DNA repeats [1-3]. In Arabidopsis, RNA-directed DNA methylation (RdDM) is responsible for de novo DNA methylation $[4,5]$. Two atypical DNA-dependent RNA polymerases IV and V (Pol IV and Pol V) are responsible for producing 24-nt small interfering RNAs (siRNAs) and long noncoding RNAs, respectively, in the RdDM pathway [6]. Pol IV produces single-stranded

\footnotetext{
*These two authors contributed equally to this work. Correspondence: Xin-Jian He

Tel: +86-10-8070-7712; Fax: +86-10-8070-7715

E-mail: hexinjian@nibs.ac.cn

Received 27 June 2014; revised 22 September 2014; accepted 29 October 2014; published online 25 November 2014
}

RNAs, which are converted into double-stranded RNAs by RNA-directed RNA polymerase 2 (RDR2) [2, 3, 6]. The double-stranded RNAs are cleaved by Dicer Like 3 (DCL3) into 24-nt siRNAs, which are loaded onto AGO4 (Argonaute 4) in the cytoplasm and subsequently transported into the nucleus for the assembly of RdDM effector complex [7-10]. Pol V generates long noncoding scaffold RNAs to recruit the RdDM effector complex [11, 12]. Pol IV and Pol V are multi-subunit RNA polymerases with NRPD1 and NRPE1 being the largest subunits of Pol IV and Pol V, respectively [13, 14]. DMS3, DRD1, and RDM1 form the DDR complex and are required for the occupancy of Pol V on chromatin $[15,16]$. In the end, DRM2 is recruited to RdDM target loci by associating with the AGO4-siRNA complex and mediates de novo DNA methylation [17].

In Arabidopsis, there are many SET domain proteins, 15 of which are related to SU(VAR)3-9, including 10 SU(VAR)3-9 homologs SUVH1-SUVH10 and 5 SU(- 
VAR)3-9 related proteins SUVR1-SUVR5 [18, 19]. The SU(VAR)3-9 homologs SUVH4/KYP, SUVH5, and SUVH6 catalyze histone H3K9 methylation and are responsible for chromatin silencing [5, 19-23]. The SUVHs contain an N-terminal YDG/SRA domain in addition to the C-terminal SET domain $[18,24]$. The SRA domain directly binds methylated DNA and is required for the function of SUVHs in H3K9 methylation [24, 25]. SUVH2 and SUVH9 are inactive histone methyltransferases and are responsible for the recruitment of Pol V to chromatin through associating with the DDR complex $[26,27]$. Different from SUVH1-SUVH10, SUVR1-SUVR5 have no SRA domain [18]. SUVR4 is an active histone methyltransferase with a preference for $\mathrm{H} 3 \mathrm{~K} 9 \mathrm{me} 1$ [28]. The N-terminal WIYLD domain of SUVR4 binds ubiquitin and facilitates the conversion of $\mathrm{H} 3 \mathrm{~K} 9 \mathrm{me} 1$ to H3K9me3 [29]. SUVR5 is involved in H3K9 methylation in vivo, although no histone methytransferase activity was detected for SUVR5 by in vitro assays [30].

Several chromatin-remodeling proteins were previously demonstrated to be involved in DNA methylation in Arabidopsis [15, 31-34]. DDM1 is an SWI2/SNF2 chromatin-remodeling protein required for maintaining DNA methylation at the whole-genome level $[31,34]$. The SNF2 chromatin-remodeling protein DRD1 can associate with DMS3 and RDM1 and forms a complex required for the recruitment of Pol V to chromatin $[15,16]$. The SNF2 chromatin-remodeling protein CLSY1 associates with Pol IV and is required for the accumulation of Pol IV-dependent siRNAs [33, 35-37].

Chromatin-remodeling proteins can also affect epigenetic silencing independently of DNA methylation. MOM1 (morpheus molecule 1), a CHD3-like chromatin-remodeling protein, is a transcriptional silencing regulator that does not affect DNA methylation [38]. MOM1 acts in transcriptional silencing independently of the RdDM pathway, however, it enhances transcriptional silencing at certain RdDM target loci $[39,40]$. MORC1 (Microrchidia 1) and MORC6/DMS11 are two members of the conserved MORC adenosine triphosphatase (ATPase) family [41, 42]. MORC1 and MORC6 are not only involved in RdDM but also are required for heterochromatin condensation [26, 41-43]. The function of MORC1 and MORC6 in heterochromatin condensation is at least partially responsible for transcriptional gene silencing in Arabidopsis. IDN2 is a canonical RdDM component that associates with its paralogs and forms a complex required for RdDM [44-46]. IDN2 was thought to be recruited to RdDM target loci by Pol V-produced long noncoding RNAs and tether the SWI/SNF chromatin-remodeling complex to the loci, thereby mediating nucleosome positioning and transcriptional gene silencing [47].
In this study, we identified SUVR2 as a regulator of transcriptional gene silencing by a forward genetic screen. The silencing of the RD29A-LUC transgene in the DNA demethylase mutant ros 1 and the silencing of endogenous transposable elements is suppressed by suvr2, while the DNA methylation of these loci is either unchanged or slightly decreased. Recently, SUVR2 was reported as a component of the RdDM pathway [5]. Our study demonstrates that SUVR2 forms a complex with its homolog SUVR1 and associates with the SNF2-related chromatin-remodeling proteins CHR19, CHR27, and CHR28, thereby mediating nucleosome positioning and transcriptional silencing. The study reveals a novel mechanism underlying transcriptional gene silencing, which may be conserved in eukaryotic organisms.

\section{Results}

\section{Identification of SUVR2 as a regulator of transcriptional} gene silencing

A robust $R D 29 A$ promoter-driven luciferase transgene (RD29A-LUC) system in the ros 1 (repressor of silencing 1) mutant background was previously developed and used for screening for cellular factors required for transcriptional gene silencing [48-51]. ROS1 encodes a DNA demethylase in Arabidopsis, and loss-of-function mutations in ROS1 lead to hypermethylation of many genomic loci $[52,53]$. The stress-inducible RD29A-LUC transgene is expressed in the wild-type background after cold treatment and the plants emit bright luminescence, whereas the transgene is silenced in ros 1 mutant plants due to hypermethylation of the transgenic RD29A promoter (Figure $1 \mathrm{~A}$ and $1 \mathrm{~B}$ ) [48]. In this transgenic line, there is another transgene, the $35 S$ promoter-driven kanamycin resistance gene NPTII (35S-NPTII), which is also highly expressed in the wild type and is silenced in ros 1 (Figure 1A and 1B) [48]. The ros 1 mutant plants harboring the $R D 29 A$ $L U C$ and $35 S-N P T I I$ transgenes were previously mutagenized by T-DNA transformation or EMS treatment and the mutagenized populations were used for screening for suppressors of ros 1 [51-54]. The mutants with reactivated expression of either RD29A-LUC and/or 35S-NPTII were subjected to map-based cloning. Most of the known RdDM components were recovered and disruption of RdDM suppressed the silencing of RD29A-LUC but not $35 S-N P T I I$ [55].

A ros $1 \# 87$ mutant was recovered in this screen, and the luminescence intensity is much higher in $\operatorname{ros} 1 \# 87$ than in rosl (Figure 1A), indicating that the silencing of the RD29A-LUC transgene is suppressed by the \#87 mutation. This effect of $\# 87$ is slightly weaker than that of the Pol V mutation nrpel (Figure 1A). The kanamycin 
A

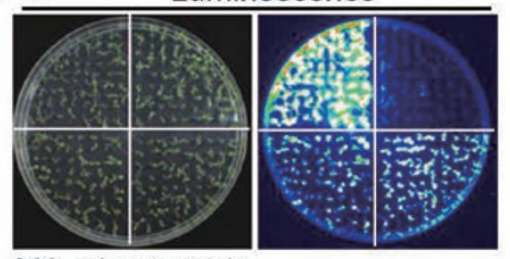

MS + kanamycin

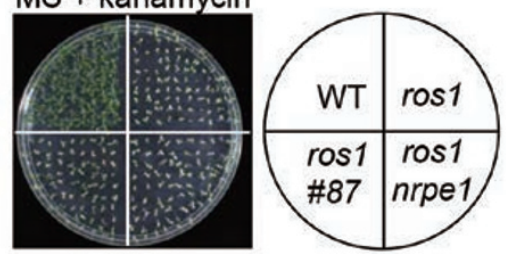

B

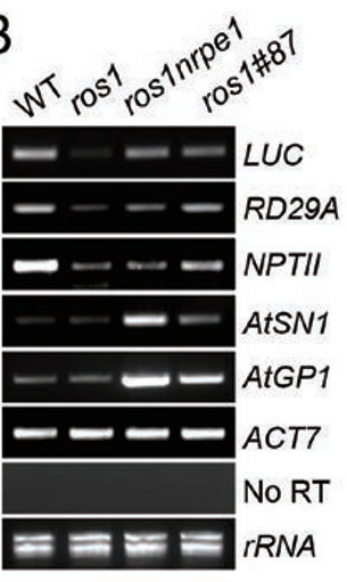

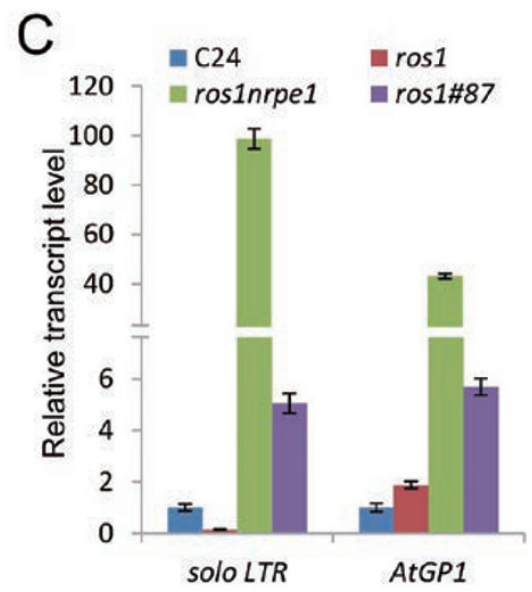

Figure 1 SUVR2 is required for transgene and transposable element silencing. (A) The luminescence phenotype and kanamycin resistance of WT, ros1, ros1\#87, and ros1nrpe1. The Arabidopsis seedlings were cold-treated at $4{ }^{\circ} \mathrm{C}$ for $2-4$ days and subjected to luminescence imaging. Kanamycin sensitivity assay was carried out on MS medium supplemental with $150 \mathrm{mg} /$ I kanamycin. (B) Semiquantitative RT-PCR was carried out to detect the RNA transcript levels of RD29A-LUC, endogenous RD29A, 35S-NPTII, and transposable elements in WT, ros1, ros1nrpe1, and ros1\#87. The actin gene ACT7 was amplified as an internal control. No RT shows amplification of ACT7 using RNA samples as templates without reverse transcription, indicating no DNA contamination in the RNA samples. (C) The RNA transcript levels of solo LTR and AtGP1 as determined by quantitative RT-PCR.

resistance is slightly higher in $\operatorname{ros} 1 \# 87$ than that in $\operatorname{ros} 1$, whereas it is comparable between ros lnrpel and ros 1 (Figure 1A). We performed RT-PCR to test the effect of the $\# 87$ mutation on the expression of RD29A-LUC. The RD29A-LUC transgene and endogenous RD29A gene are silenced in ros 1 but reactivated in $\operatorname{ros} 1 \# 87$, with ros 1nrpel serving as a positive control (Figure 1B). The $35 S-N P T I I$ transgene is silenced in ros 1 and is partially reactivated in ros $1 \# 87$ but is not reactivated in ros 1nrpel (Figure 1B), which is consistent with the kanamycin resistance phenotypes of the mutants as seen in Figure 1A. The transposable elements AtSN1, AtGP1, and solo LTR are typical RdDM target loci [9, 56, 57]. We found that the transcript levels of these loci are increased in roslnrpe1 and to a lesser extent in ros $1 \# 87$ (Figure 1B and 1C). These data suggest that the $\# 87$ mutation affects transcriptional gene silencing at RdDM target loci.

We performed map-based cloning using the F2 segregating population from a cross between the ros $1 \# 87$ mutant in the C24 background and the ros 1-4 mutant (Salk_045303) in the Col-0 background. The \#87 mutation was mapped to a $\sim 1 \mathrm{Mb}$ region on chromosome 5 (Supplementary information, Figure S1A). We sequenced the candidate genes in this region and found a G-to-A mutation at the splice receptor site of the AT5G43990 (SUVR2) second intron (Supplementary information, Figure S1B). The mutation causes a frame shift in the SUVR 2 coding sequence in the ros1\#87 mutant (Supple- mentary information, Figure S1C). SUVR2 is a member of the Drosophila SU(VAR)3-9 related proteins and contains three conserved domains, which are the N-terminal WIYLD domain, the C-terminal Pre-SET and SET domains (Supplementary information, Figure S1B). To determine whether the mutation in SUVR2 is responsible for the silencing of the RD29A-LUC transgene, we transformed a construct harboring the full-length SUVR2 genomic sequence into the ros $1 \# 87$ mutant and found that the silencing defect of RD29A-LUC in ros $1 \# 87$ is complemented by the $S U V R 2$ construct in several individual SUVR2 transgenic lines (Supplementary information, Figure S1D). The results suggest that the G-to-A mutation in SUVR2 is responsible for the defective silencing phenotype of $\operatorname{ros} 1 \# 87$. Thus, $\# 87$ is thereafter referred to as suvr2.

SUVR2 has both RdDM-dependent and-independent roles in transcriptional gene silencing

We performed locus-specific bisulfite sequencing to determine whether the effect of suvr2 on transcriptional gene silencing is due to a reduction in DNA methylation. Our data demonstrated that the DNA methylation levels of the transgenic and endogenous RD29A promoters are low in wild-type plants, whereas they are obviously increased in rosl (Figure 2A). The DNA methylation levels of the transgenic and endogenous RD29A promoters are either not affected or slightly decreased in ros 1 suvr 2 
compared to those in ros 1 (Figure 2A). As reported previously [54], DNA methylation at the promoter of RD29A-LUC is markedly reduced by the RdDM mutation nrpe1. Although suvr2 has no significant effect on DNA methylation at the transgenic RD29A promoter, the silencing of $R D 29 A-L U C$ is markedly released by suvr2 (Figure 1A and 1B). These results suggest that the action of SUVR2 in the silencing of RD29A-LUC and endogenous $R D 29 A$ is at least partially independent of DNA methylation. AtSN1, solo LTR and MEA-ISR are RdDM target loci $[9,57,58]$. Our result indicated that DNA methylation of the three loci is markedly decreased by the Pol IV mutation nrpd1 in the ros Inrpd1 mutant (Figure 2B). In the ros 1suvr2 mutant, DNA methylation is slightly decreased at MEA-ISR and solo LTR but not at AtSN1 (Figure 2B) even though the transcript level of AtSN1 is clearly increased (Figure 1B). Thus, the involvement of SUVR2 in the silencing of AtSN1 is independent of DNA methylation. These data suggest that suvr2 affects DNA methylation at a subset of RdDM target loci, but the defective transcriptional silencing caused by suvr 2 is not necessarily coupled with a decrease in DNA methylation. Thus, the involvement of SUVR2 in transcriptional gene silencing is likely partially through a DNA methylation-independent mechanism.

Although the suvr2 mutation affects DNA methylation at the RdDM target loci MEA-ISR and solo LTR, the effect of suvr2 is much weaker than that of the canonical RdDM mutation nrpdl (Figure 2B). To confirm the effect of suvr 2 on DNA methylation, we tested DNA methylation at another RdDM target locus, IGN23. For analysis of the IGN23 DNA methylation, genomic DNA was digested by the DNA methylation-sensitive restriction enzyme HaeIII followed by quantitative PCR. The result indicated that the DNA methylation level of IGN23 is significantly reduced in ros 1 suvr 2 relative to ros 1 (Supplementary information, Figure S2). A full-length SUVR2 construct was transformed into ros 1 suvr 2 to determine whether the SUVR2 transgene rescues the defect in DNA methylation. In two individual SUVR2 transgenic lines, the IGN23 methylation level is not only higher than that in ros 1suvr2 but also in the wild type and ros 1 (Supplementary information, Figure S2). These results demonstrates that SUVR2 is necessary for DNA methylation at a subset of RdDM target loci.

Small RNA deep sequencing was performed to de-
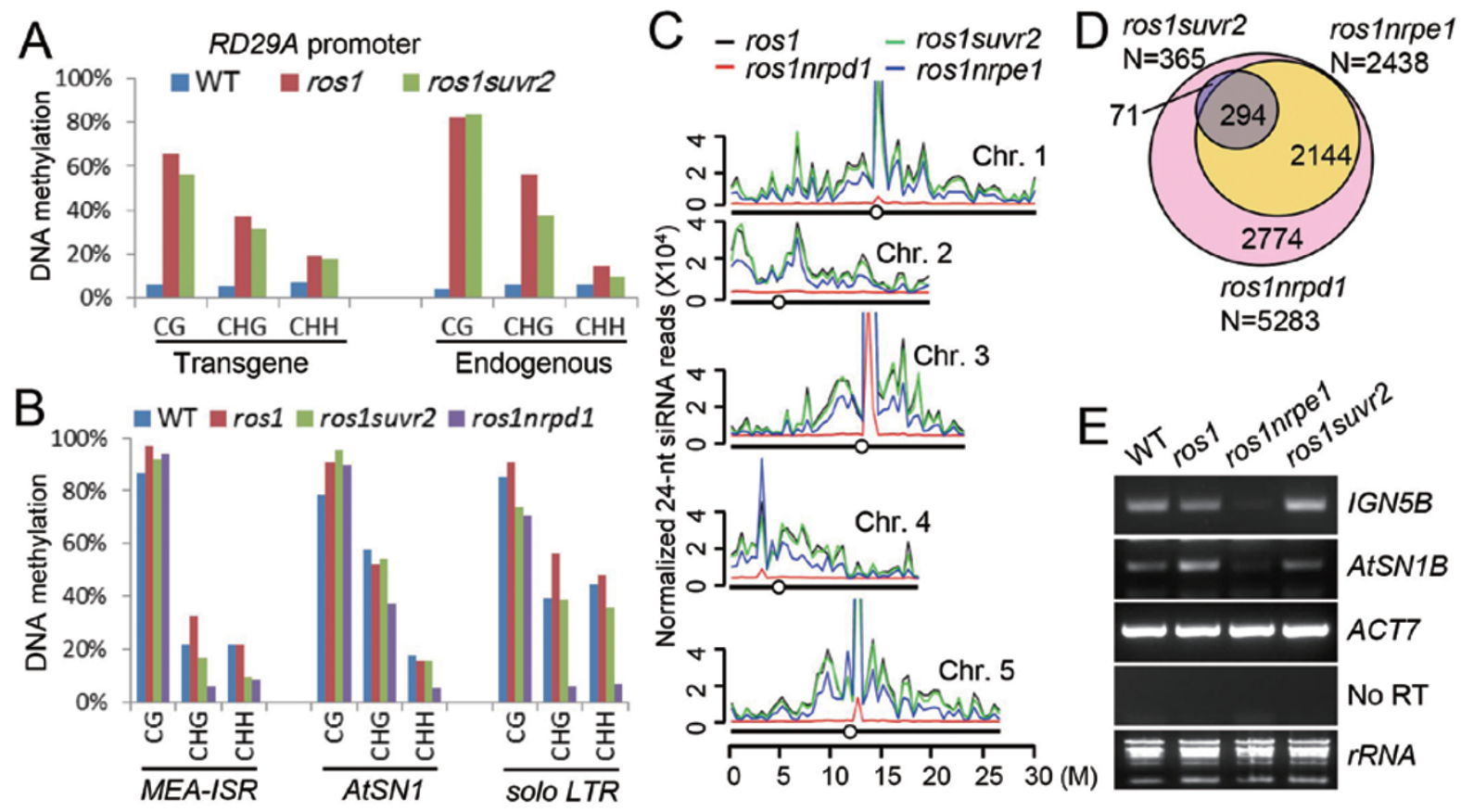

Figure 2 The function of SUVR2 in transcriptional silencing is partially independent of RdDM. (A, B) DNA methylation was determined by bisulfite sequencing at the promoters of transgenic RD29A-LUC and endogenous RD29A, MEA-ISR, AtSN1, and solo LTR. The percentages of $\mathrm{CG}, \mathrm{CHG}$, and $\mathrm{CHH}$ methylation are separately shown. (C) Plots indicate the distribution of Pol IV-dependent 24-nt siRNA across the five Arabidopsis chromosomes in ros1, ros1suvr2, ros1nrpd1, and ros1nrpe1. (D) Venn diagram shows the numbers of affected 24-nt siRNA regions and their overlaps in ros1suvr2, ros1nrpd1, and ros1nrpe1 relative to ros1. (E) Accumulation of Pol V-produced noncoding RNAs from AtSN1B and IGN5B was detected by semiquantitative RT-PCR. The dependence of the noncoding RNAs on Pol V was shown in ros1nrpe1 as a control. 
termine whether suvr2 affects transcriptional silencing by attenuating Pol IV-produced 24-nt siRNAs. The deep sequencing data indicated that the Pol IV mutation nrpdl results in a severe reduction of 24-nt siRNAs throughout all 5 chromosomes while the Pol V mutation nrpel leads to an obvious reduction of 24-nt siRNAs at two arms of each chromosome but has little effect on centromeric regions (Figure 2C). However, the overall abundance of Pol IV-dependent 24-nt siRNAs in ros 1suvr2 is similar to that in ros 1 (Figure 2C, Supplementary information, Table S1). We counted the numbers of 24-nt siRNA regions, in which the 24-nt siRNAs are downregulated in ros1suvr2, ros 1nrpd1, and ros 1nrpel compared to ros 1 (Figure 2C, Supplementary information, Table S1). 24nt siRNAs from 5283 siRNA regions are significantly downregulated in ros 1nrpd1 compared to ros 1, among which siRNAs from 2438 regions are also downregulated in ros 1nrpel (Figure 2D, Supplementary information, Table S1). Different from the RdDM mutants nrpd1 and nrpe1, suvr 2 only affects 365 24-nt siRNA regions, which are $7 \%$ of the NRPD1 targets. 294 of the 365 regions overlap with the NRPE1 targets, while 71 overlap with NRPD1 targets but are independent of NRPE1 (Figure 2D). Our data demonstrate that the effect of suvr2 on 24-nt siRNA accumulation is much more limited than that of nrpe1, which is different from a previous study reporting that suvr2 and nrpel have a similar effect on 24-nt siRNA accumulation [5].

Pol V produces long noncoding RNAs, which are responsible for the assembly of RdDM effector complex

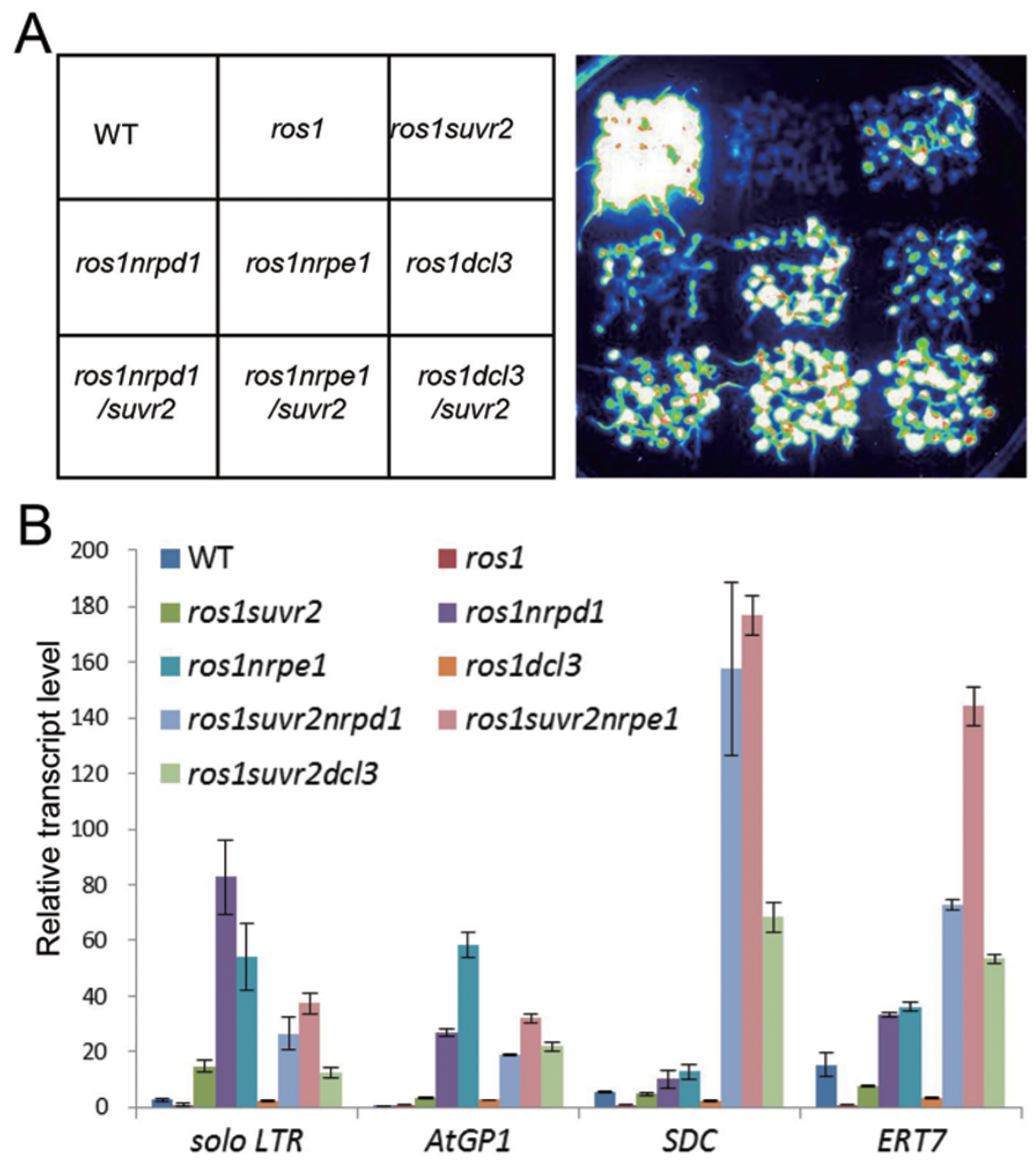

Figure 3 The genetic relationship between SUVR2 and RdDM components. (A) The expression of RD29A-LUC transgene is indicated by luminescence imaging. The luminescence images are shown for the wild type, ros1, ros1suvr2, ros1nrpd1, ros1nrpe1, and ros1dc/3, as well as for the indicated mutants in which the suvr2 mutation was combined with each of the RdDM mutations. (B) The transcript levels of the endogenous RdDM target loci solo LTR, AtGP1, SDC, and ERT7 were determined by quantitative RT-PCR. The experiments were biologically repeated for three times. 
[11]. We tested whether suvr2 affects Pol V-produced noncoding RNAs at the $I G N 5 B$ and $A t S N 1 B$ sites. The $I G N 5 B$ and $A t S N I B$ transcripts are blocked by the Pol V mutation nrpel but are not affected by suvr2 (Figure 2E), indicating that SUVR2 is not required for Pol V transcription. The result suggests that the action of SUVR2 is either at a step downstream of Pol V transcription in the RdDM pathway or independent of RdDM.

To investigate the relationship between SUVR2 and the RdDM pathway, we determined whether SUVR2 interacts with major RdDM components. Because SUVR2 is not required for the accumulation of most Pol IV-dependent siRNAs (Figure 2C and 2D, Supplementary information, Table S1), it is unlikely involved in Pol IV-dependent siRNA biogenesis at the early steps of the RdDM pathway. Thus, only the downstream RdDM components NRPE1, AGO4, DMS3, RDM1, KTF1, and DRM2 were selected for the interaction assay. By co-IP, we found that none of these RdDM components interact with SUVR2 (Supplementary information, Figure S3A$\mathrm{S} 3 \mathrm{~F})$. We further performed nuclear coimmunolocalization assays to determine whether SUVR2 colocalizes with DRM2, NRPE1, or KTF1 in the nucleus. The results indicated that SUVR2 forms a few condensed foci in the nucleus but does not significantly overlap with the signals of DRM2, NRPE1, and KTF1 (Supplementary information, Figure S4A-S4C).

Many RdDM components were identified from the ros 1 suppressor screen, including the core components NRPD1, NRPE1 and DCL3 (Figure 3A). To examine the genetic relationship between SUVR2 and the RdDM pathway, ros1suvr2 was crossed to ros1nrpd1, roslnrpe1, and ros $1 d c l 3$ to combine suvr 2 with RdDM mutations. Luminescence images show that the expression of the RD29A-LUC transgene is suppressed in ros 1 but reactivated by suvr 2 , nrpdl, nrpel and $d c l 3$ (Figure 3A). The RD29A-LUC expression level in ros 1 suvr 2 is comparable to that in roslnrpd1 and rosldcl3 but weaker than that in ros 1nrpel (Figure 3A). In ros 1nrpd1suvr2 and rosldcl3suvr2, the RD29A-LUC expression is synergistically enhanced as compared with that in roslnrpd1, ros $1 d c l 3$, and roslsuvr2 (Figure 3A). In contrast, the RD29A-LUC expression is less enhanced in ros 1nrpelsuvr2 relative to that in ros 1nrpel (Figure 3A). We performed quantitative RT-PCR to determine the transcript levels of the endogenous RdDM target loci solo LTR, AtGPl, SDC, and ERT7 (Figure 3B). The results indicated that the silencing of all these loci is relieved by suvr 2, nrpd1, nrpe1, and $d c l 3$ (Figure 3B). At these RdDM target loci, the effect of suvr2 and $d c l 3$ is comparable but is weaker than that of nrpdl and nrpel. The weak effect of $d c l 3$ is consistent with the functional redundancy between DCL3 and its close homologs DCL2 and DCL4 in RdDM [59]. The transcript levels of solo LTR and AtGP1 are not increased by suvr2 in ros 1suvr2nrpd1, ros1suvr2nrpe1, but are increased by suvr2 in ros 1 suvr $2 d c l 3$ (Figure 3B). Meanwhile, at $S D C$ and ERT7 sites, the effect of suvr2 is synergistic with that of all the RdDM mutations (Figure 3B), suggesting that the involvement of SUVR2 in the silencing of $S D C$ and ERT7 is at least partially through an RdDM-independent pathway. These results suggest that the interplay between SUVR2 and the RdDM pathway is in a locus-specific manner.

We performed RNA-seq to compare the effect of suvr 2 and nrpel on the transcript levels of genes and TEs at the whole-genome level. $5.9 \times 10^{7}, 5.6 \times 10^{7}$, and $5.0 \times 10^{7}$ reads were obtained from the libraries of $\operatorname{ros} 1$, ros 1nrpe1, and ros 1suvr2, respectively (Supplementary information, Table S2). More than $97 \%$ of the reads were mapped to the Arabidopsis genome sequence. From the RNA-seq data, we found that many genes and TEs are coregulated by suvr2 and nrpel (Figure 4A-4D, Supplementary information, Tables S3-S6). The numbers of upregulated TEs in suvr 2 and nrpel are much higher than those of downregulated ones (Figure 4B and 4D), which is consistent the function of SUVR2 and NRPE1 in the silencing of TEs. The RNA-seq data indicated that 163 genes and 59 TEs are significantly upregulated by suvr 2 (Figure 4C and 4D, Supplementary information, Tables S3 and S5). Among them, 32.9\% (54/163) of genes and $33.9 \%(20 / 59)$ of TEs overlap with the upregulated genes and TEs caused by nrpe1, respectively (Figure 4C and 4D, Supplementary information, Tables S3-S6). The overlapping of upregulated genes and TEs in suvr2 and nrpel is significantly higher than expected by chance $(P$ $<0.01$ ), suggesting that SUVR2 can act at a subset of RdDM target loci. Additionally, about two-third of upregulated genes and TEs caused by suvr 2 do not overlap with those caused by nrpel (Figure 4A-4D, Supplementary information, Tables S3-S6). These results support the inference that SUVR2 has both RdDM-dependent and -independent roles in transcriptional gene silencing.

We randomly selected the genes and TEs that are specifically upregulated by suvr 2 as determined by our RNA-seq experiment (Figure 4A-4D, Supplementary information, Tables S3-S6) to confirm their expression by quantitative RT-PCR (Figure 4E and 4F). The results suggest that most of the selected loci $(7 / 8$ for genes and $6 / 7$ for TEs) are significantly up-regulated by suvr 2 but either not affected or weakly affected by nrpel (Figure $4 \mathrm{E}$ and $4 \mathrm{~F})$. Because the nrpel mutation was thought to completely block the RdDM pathway, the finding of the SUVR2-specific target loci demonstrates that SUVR2 has an RdDM-independent role in transcriptional gene 

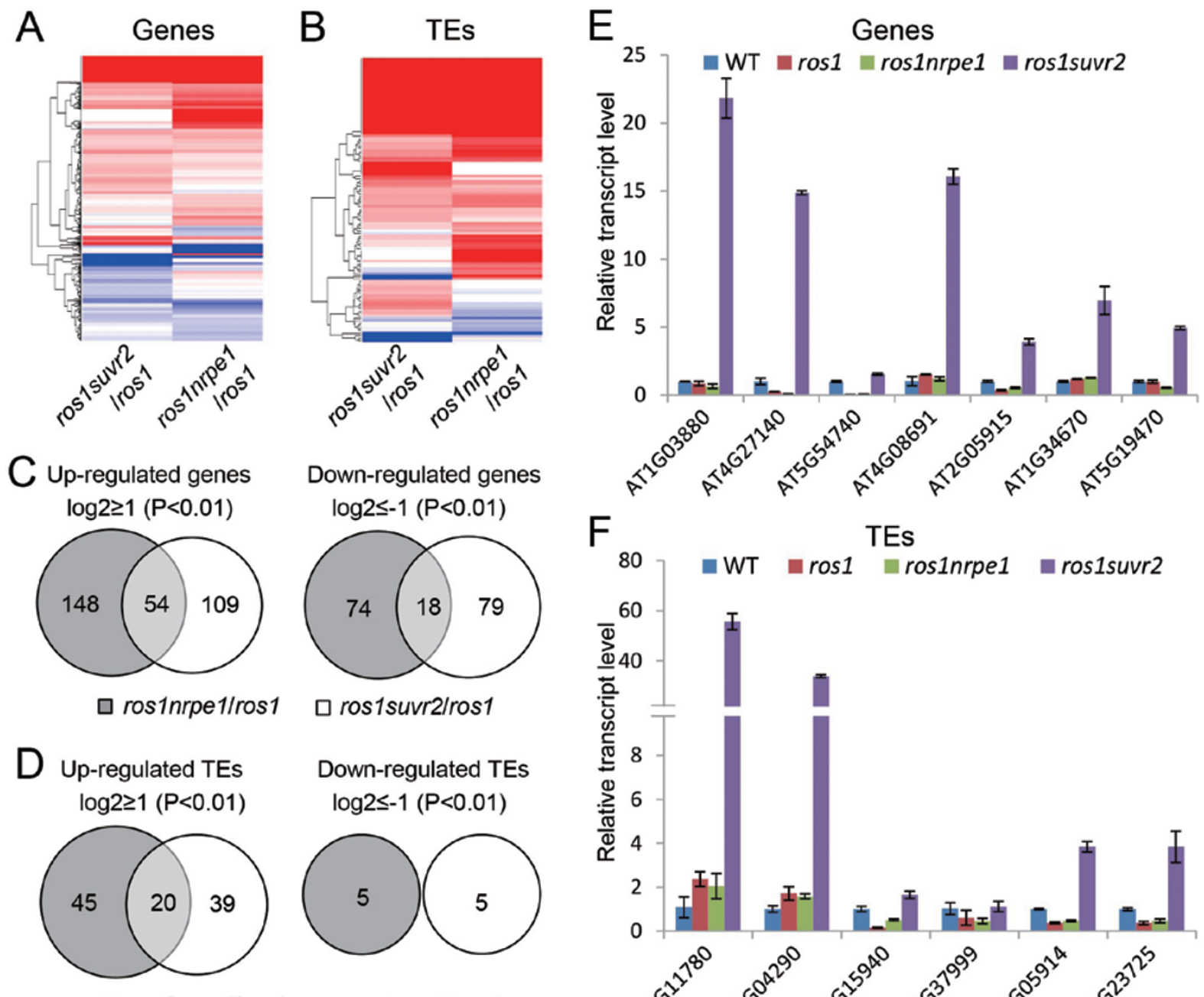

ros1nrpe1/ros1
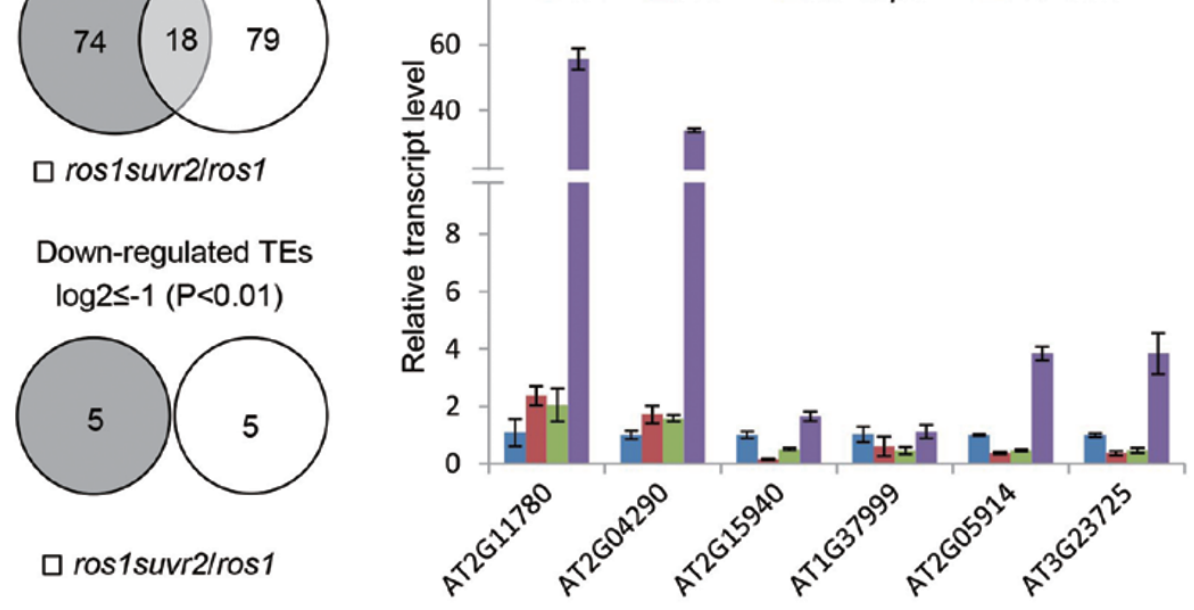

Figure 4 The effect of suvr2 and nrpe1 on RNA transcript levels as determined by RNA-seq. (A, B) Heat maps indicate differentially expressed genes (A) and TEs (B) in ros1suvr2 and ros1nrpe1 relative to ros1. Red and blue bars represent upregulated and downregulated genes or TEs, respectively. (C, D) Differentially expressed genes (C) or TEs (D) were compared between ros1suvr2 and ros1nrpe1. Differentially expressed genes or TEs were defined when $\log _{2}$ (fold changes of normalized reads) $\geq 1$ or $\leq-1$ and $P$ value $<0.01$. (E, F) Quatitative RT-PCR was performed to confirm SUVR2-specific target genes (E) and TEs $(F)$ identified by RNA-seq.

silencing in addition to its role in the RdDM pathway.

The function of SUVR2 in transcriptional gene silencing is independent of histone H3K9 methylation

The above bisulfite sequencing result suggests that DNA methylation at the promoters of RD29A-LUC and endogenous $R D 29 A$ is not significantly affected by suvr2 (Figure 2A). We performed a ChIP assay to determine the effect of suvr2 on the repressive histone mark $\mathrm{H} 3 \mathrm{~K} 9 \mathrm{me} 2$. The result indicated that the H3K9me2 levels at the transgenic and endogenous $R D 29 \mathrm{~A}$ promoters are increased by $\operatorname{ros} 1$ (Figure 5A and 5B). This effect of ros 1 is consistent with previous reports $[60,61]$. In the ros 1 mutant background, the enrichment of $\mathrm{H} 3 \mathrm{~K} 9$ me 2 on the transgenic and endogenous $R D 29 \mathrm{~A}$ promoters is clearly reduced by nrpel (Figure 5A and $5 \mathrm{~B}$ ), which is consistent with the previous reports showing that nrpel affects $\mathrm{H} 3 \mathrm{~K} 9 \mathrm{me} 2$ at RdDM target loci $[11,57]$. However, suvr2 has no effect on $\mathrm{H} 3 \mathrm{~K} 9 \mathrm{me} 2$ at the transgenic and endogenous $R D 29 \mathrm{~A}$ promoters (Figure $5 \mathrm{~A}$ and $5 \mathrm{~B}$ ). Moreover, we found that the enrichment of $\mathrm{H} 3 \mathrm{~K} 9 \mathrm{me} 2$ at the endogenous RdDM target loci solo LTR and IGN5 is reduced by nrpel but not by suvr 2 (Figure 5C and 5D). These results suggest that the function of SUVR2 in tran- 

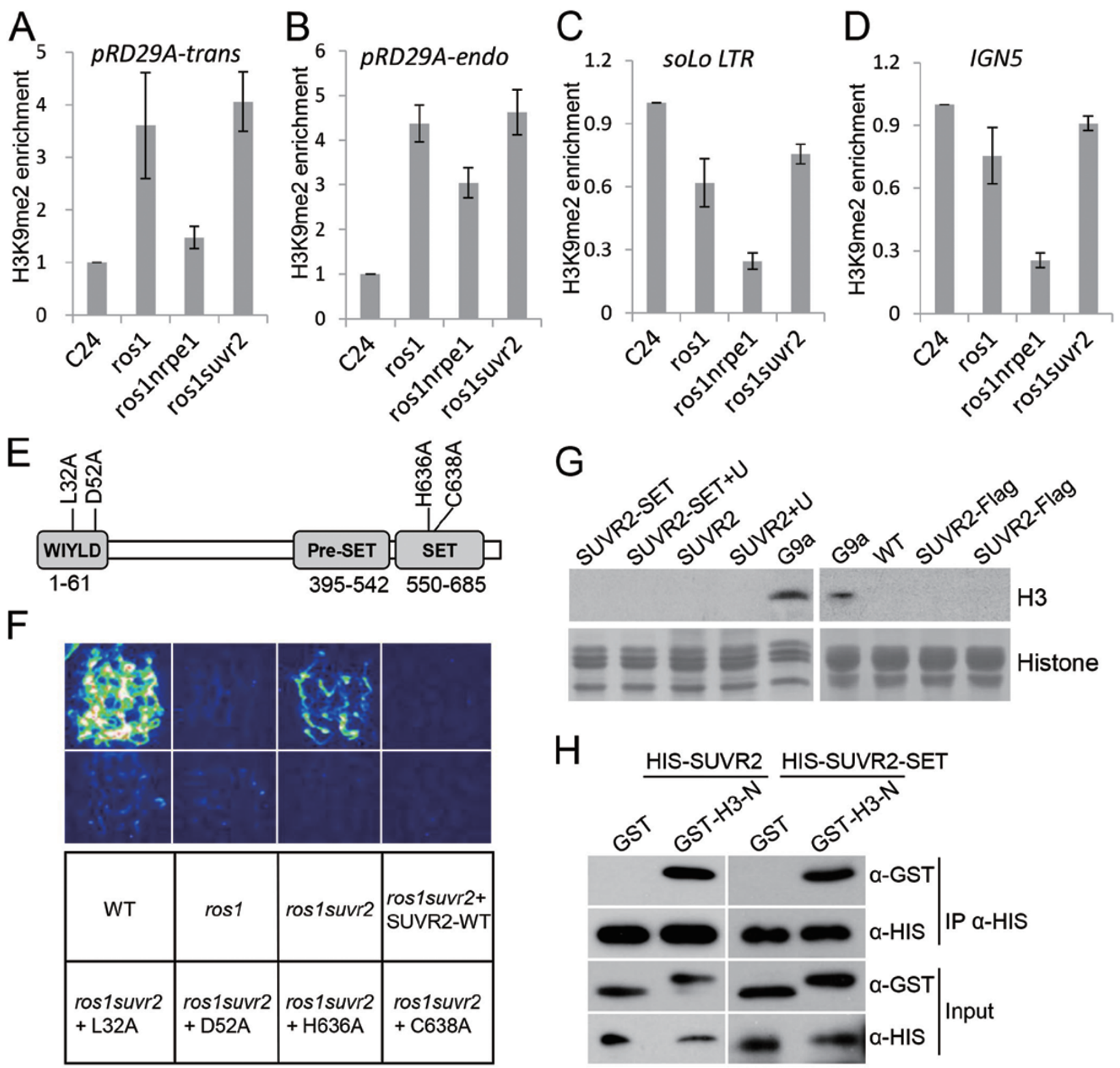

Figure 5 SUVR2 is involved in transcriptional gene silencing independently of the histone H3K9 methyltransferase activity. (A-D) The H3K9me2 levels at the promoters of RD29A-LUC (A) and endogenous RD29A (B), solo LTR (C), and IGN5 (D) were determined by ChIP-PCR in the wild type, ros1, ros1suvr2, and ros1nrpe1. The protein-coding gene ACT7 that has a low H3K9me2 level was used as an internal control. (E) The diagram of the SUVR2 protein indicates that SUVR2 contains three conserved domains: WIYLD, Pre-SET, and SET. The conserved residues that have been subjected to point mutation in this study are shown. (F) The complementation test by using wild-type and mutated SUVR2 transgenes. The constructs harboring wild-type and mutated SUVR2 sequences were introduced into ros1suvr2. The luminescence image indicates the expression of RD29A-LUC. In the mutated SUVR2 sequences, L32 and D52 in the WIYLD domain, and H636 and C638 in the SET domain are mutated to alanine. (G) The SUVR2 protein was purified from bacteria and Arabidopsis and was used in the in vitro histone methyltransferase activity assay. The human SU(VAR)3-9 homolog G9a that shows active histone H3K9 methylation was used as a positive control. "U" is ubiquitin. (H) The interaction of SUVR2 with the histone H3 peptide as determined by pull-down assay. The full-length SUVR2 and the SUVR2 fragment containing the PreSET and SET domains were bacterially expressed in fusion with the His tag. The N-terminus of the histone H3 was expressed in fusion with the GST tag in bacteria. 
scriptional gene silencing is independent of $\mathrm{H} 3 \mathrm{~K} 9 \mathrm{me} 2$. Moreover, we performed $\mathrm{H} 3 \mathrm{~K} 9 \mathrm{me} 3 \mathrm{ChIP}$ to determine the $\mathrm{H} 3 \mathrm{~K} 9$ me3 levels at the RdDM target loci solo LTR, $I G N 5$, and IGN23. The results indicated that the enrichment of $\mathrm{H} 3 \mathrm{~K} 9 \mathrm{me} 3$ relative to input at the RdDM target loci ( $\sim 0.03 \%-0.05 \%$ at solo LTR, $\sim 0.015 \%-0.025 \%$ at $I G N 5$, and $\sim 0.01 \%$ at $I G N 23)$ is much lower than that at the protein-coding gene $A C T 2(\sim 0.09 \%)$ (Supplementary information, Figure S5), which is consistent with the previous studies reporting that $\mathrm{H} 3 \mathrm{~K} 9 \mathrm{me} 3$ is preferentially associated with euchromatic protein-coding genes but not with heterochromatic transposable elements $[62,63]$. The H3K9me3 levels at solo LTR, IGN5, and IGN23 are not reduced by nrpe1 and suvr2 (Supplementary information, Figure S5). Thus, H3K9me3 is unlikely to play a role in transcriptional gene silencing at transposable elements targeted by SUVR2.

SUVR2 is a SU(VAR)3-9-related protein and contains three annotated domains, which are the WIYLD domain, the Pre-SET domain, and the SET domain (Figure 5E). The WIYLD domain is conserved in the SUVR family in Arabidopsis [28]. The WIYLD domain of SUVR4 binds ubiquitin and affects the histone methyltransferase activity of SUVR4 [29]. The SUVR4 residue D74 in the WIYLD domain is required for the binding of SUVR4 to ubiquitin [29]. The SUVR2 residue D52 corresponds to the SUVR4 residue D74 (Figure 5E, Supplementary information, Figure S6A). The SET domain is conserved in SU(VAR)3-9 homologs (Supplementary information, Figure S6B). Previous structural and biochemical studies indicated that critical residues in the SET domain of SU(VAR)3-9 homologs are essential for histone H3K9 methyltransferase activity [20,64]. The SUVR2 residues H636 and C638 correspond to the essential catalytic residues in the SU(VAR)3-9 family proteins (Figure 5E, Supplementary information, Figure S6B).

To determine whether the critical residues in the WIYLD domain and the SET domain are required for the function of SUVR2 in transcriptional gene silencing, we generated SUVR2 constructs harboring the WIYLD domain mutations L32A and D52A, and the SET domain mutations H636A and C638A, and transformed the constructs into ros 1 suvr 2 to investigate whether the mutated SUVR2 can rescue the defective transcriptional gene silencing of ros 1 suvr 2 . Luminescence imaging indicated that the silencing of $R D 29 A-L U C$ was restored by the four mutated SUVR2 sequences, as well as by the wild-type SUVR2 sequence in ros1suvr2 (Figure 5F). Quantitative RT-PCR indicated that the silencing of both $R D 29 A-L U C$ and endogenous $R D 29 A$ is not affected by the mutations in SUVR2 (Supplementary information, Figure S7). Moreover, we checked the transcript level of the endogenous transposable element $A t G P 1$ and found that the silencing of AtGP1 is restored by both wild-type and mutated SUVR2 transgenes (Supplementary information, Figure S7). These results suggest that the SUVR2 mutations do not affect the function of SUVR2 in transcriptional gene silencing. Thus, none of the four sites that were mutated in the WIYLD and SET domains is required for the function of SUVR2 in transcriptional gene silencing, suggesting that the involvement of SUVR2 in transcriptional gene silencing is independent of ubiquitin binding and histone methyltransferase activity.

Histone methyltransferase activity was not detected for SUVR1 and SUVR2 in a previous study [28]. We tested the histone methyltransferase activity of SUVR2 using the SUVR2 proteins purified from both bacteria and Arabidopsis. Neither the full-length SUVR2 nor the SET domain of SUVR2 can methylate core histones from calf thymus, whereas the human SU(VAR)3-9 homolog, G9a, can effectively methylate the histones (Figure 5G, left panel). Since addition of ubiquitin can affect the histone methyltransferase activity of SUVR4 [29], we examined whether ubiquitin may stimulate the histone methyltransferase activity of SUVR2. The results showed that no activity was detected even when ubiquitin was added (Figure 5G, left panel). The histone methyltransferase activity of SUVR2 was not detected even when SUVR2 was expressed in Arabidopsis (Figure 5G, right panel). These results suggest that SUVR2 is not an active histone methyltransferase and is involved in transcriptional gene silencing through an unknown mechanism.

Although SUVR2 fails to methylate histone $\mathrm{H} 3$ at lysine 9, it is still possible that SUVR2 is capable of binding the histone $\mathrm{H} 3$ peptide. The full-length SUVR2 and the truncated SUVR2 containing the PreSET and SET domains were purified from bacteria and used to test whether they bind the histone H3 peptide. The results indicated that both full-length and truncated SUVR2 proteins bind the $\mathrm{N}$-terminus of $\mathrm{H} 3$ as determined by the in vitro pull-down assay (Figure $5 \mathrm{H}$ ), suggesting that the PreSET and SET domains of SUVR2 may associate with chromatin through binding to $\mathrm{H} 3$.

\section{SUVR2 forms a complex with SUVR1 and associates with CHR19, CHR27, and CHR28}

To understand how SUVR2 is involved in transcriptional gene silencing, we tried to isolate SUVR2-interacting proteins by affinity purification of SUVR2-Flag in SUVR2-Flag transgenic plants. Mass spectrometric data indicated that SUVR2-Flag copurified with its close homolog SUVR1 and three SNF2 ATP-dependent chromatin-remodeling proteins including CHR 19, CHR27 and CHR28 (Table 1, Supplementary information, Table 
Table 1 Mass spectrometric analyses of SUVR2, SUVR1, CHR19, and CHR27 affinity purification

\begin{tabular}{llllllllll}
\hline \multirow{2}{*}{ AGI code } & \multirow{2}{*}{ Protein } & \multicolumn{3}{c}{ SUVR2-Flag } & \multicolumn{2}{c}{ SUVR1-Flag } & \multicolumn{2}{c}{ CHR19-Myc } & \multicolumn{2}{c}{ CHR27-Myc } \\
\cline { 3 - 10 } & & $\begin{array}{l}\text { Mascot } \\
\text { score }\end{array}$ & $\begin{array}{l}\text { Unique } \\
\text { peptides }\end{array}$ & $\begin{array}{l}\text { Mascot } \\
\text { score }\end{array}$ & $\begin{array}{l}\text { Unique } \\
\text { peptides }\end{array}$ & $\begin{array}{l}\text { Mascot } \\
\text { score }\end{array}$ & $\begin{array}{l}\text { Unique } \\
\text { peptides }\end{array}$ & $\begin{array}{l}\text { Mascot } \\
\text { score }\end{array}$ & $\begin{array}{l}\text { Unique } \\
\text { peptides }\end{array}$ \\
\hline AT5G43990 & SUVR2 & 18282 & 46 & 1232 & 11 & 217 & 3 & 282 & 3 \\
AT1G04050 & SUVR1 & 814 & 10 & 5199 & 34 & 0 & 0 & 0 & 0 \\
AT2G02090 & CHR19/ETL1 & 804 & 11 & 0 & 0 & 8937 & 57 & 0 & 0 \\
AT3G20010 & CHR27 & 285 & 5 & 0 & 0 & 0 & 0 & 2716 & 35 \\
AT1G50410 & CHR28 & 98 & 3 & 0 & 0 & 0 & 0 & 0 & 0 \\
\hline
\end{tabular}

S7). To confirm the interaction of SUVR2 with SUVR1, CHR19, and CHR27, we generated transgenic plants harboring SUVR1-Flag, CHR19-Myc, or CHR27-Myc transgene, and used these plants to perform affinity purification of SUVR1-Flag, CHR19-Myc, and CHR27-Myc, respectively. Mass spectrometric analysis indicated that SUVR2 is copurified with SUVR1-Flag, CHR19-Myc, and CHR27-Myc (Table 1, Supplementary information, Table S7). Co-IP experiments demonstrated that SUVR2 interacts with SUVR1 as well as with itself (Figure 6A and $6 \mathrm{~B}$ ). We performed GST pull-down assays to determine whether SUVR1 and SUVR2 may directly interact with each other. The results indicated that bacterially expressed GST-SUVR1 can pull down both His-SUVR2 and His-SUVR1 (Figure 6C and 6D), confirming that SUVR1 is able to form a complex with SUVR1 or SUVR2. Unexpectedly, we found that GST-SUVR2 and His-SUVR2 is unable to pull down each other (Supplementary information, Figure S8A and S8B). However, the interaction between two SUVR2 proteins was confirmed by a yeast two-hybrid assay (Figure $6 \mathrm{E}$ and $6 \mathrm{~F}$ ). It is possible that some modifications that are required for the SUVR2-SUVR2 interaction are absent in the bacterially expressed SUVR2.

To identify the domain that is required for the SUVR2-SUVR2 interaction, we produced constructs harboring truncated SUVR2 versions and separately introduced the constructs into yeast for two-hybrid assays (Figure $6 \mathrm{E}$ and $6 \mathrm{~F})$. The results indicated that the truncated sequences containing both PreSET and SET domains can interact with SUVR2 and the absence of either domain causes a loss of the interaction (Figure 6E and 6F, Supplementary information, Figure S9), suggesting that the PreSET and SET domains are not only necessary but also sufficient for the interaction between two SUVR2 proteins.

We introduced both SUVR2-Flag and CHR19-Myc into Arabidopsis to determine the interaction between SUVR2 and CHR19 by co-IP. The result demonstrated that SUVR2 interacts with CHR19 (Figure 6G). In gel filtration, SUVR1 and SUVR2 were largely coeluted in the fractions of $>440 \mathrm{KDa}$ (Figure $6 \mathrm{H}$ ), indicating that the two proteins exist in a tight complex in vivo. SUVR2 and CHR19 show overlapping but different elution patterns in gel filtration (Figure 6H), suggesting that the interaction between SUVR2 and CHR19 is probably spatiotemporally specific in vivo.

Nuclear coimmunolocalization assays were performed to test whether SUVR2 colocalizes with SUVR1 and CHR19. SUVR2 shows two nuclear localization patterns with one forming condensed foci $(50 / 112)$ and the other showing diffused signals (62/112) (Figure 7A). The condensed SUVR2 foci are as large as heterochromatin foci that are indicated by DAPI staining and the H3K27me1 mark (Figure 7A). The condensed SUVR2 foci are close to but not colocalized with the heterochromatin foci (Figure 7A), which is consistent with the role of SUVR2 in silencing RdDM target loci rather than genomic loci at heterochromatin regions. When SUVR2 is present as condensed foci in the nucleus, both SUVR1 and CHR19 signals fully overlap with SUVR2 signal (Figure 7B), which supports the interaction of SUVR2 with SUVR1 and CHR19. However, we did not find that the SUVR2 signals overlap with the signals of the RdDM components DRM2, NRPE1, and KTF1 (Supplementary information, Figure S4A-S4C). The data are consistent with the finding that SUVR2 forms a tight complex with SUVR1 and associates with the chromatin-remodeling protein CHR19 (Figure 6A-6H).

CHR19, CHR27, CHR28, and SUVR1 are required for transcriptional gene silencing

Because SUVR2 associates with the chromatin-remodeling proteins CHR19, CHR27, and CHR28, it is possible that the three chromatin-remodeling proteins function in transcriptional gene silencing as SUVR2 does. Quantitative RT-PCR indicated that the silencing of well-studied RdDM target loci such as solo LTR, AtGP1, and $S D C$ is suppressed in the suvr 2 single mutant (Figure $8 \mathrm{~A}$ ), which is consistent with the observation in the ros- 
A

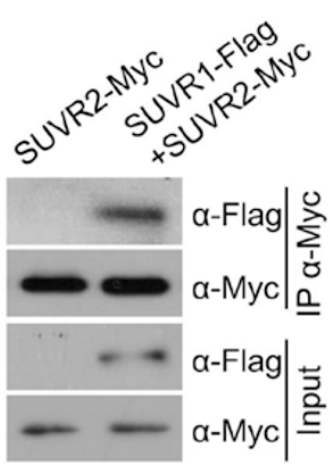

B

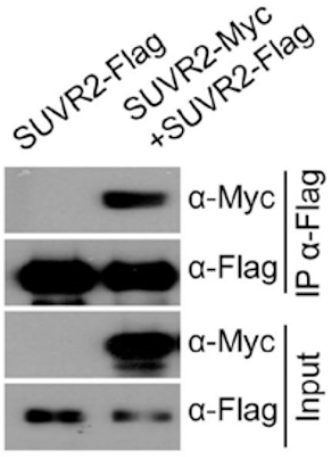

G

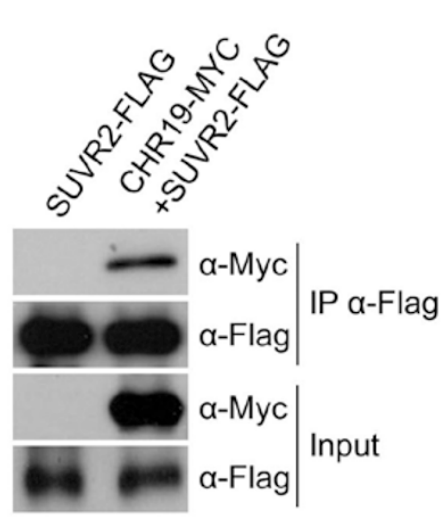

C

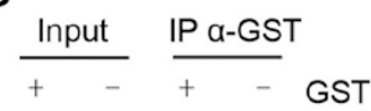

$-\quad+\quad-\quad+$ GST-SUVR1

$+\quad+\quad+\quad+$ HIS-SUVR2

$\Longrightarrow$ GST

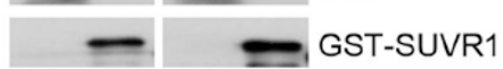

$\longrightarrow$ HIS-SUVR2

D

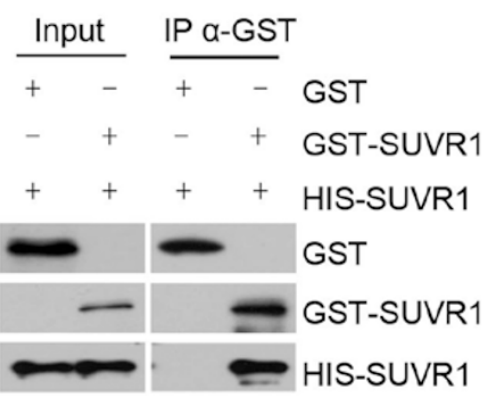

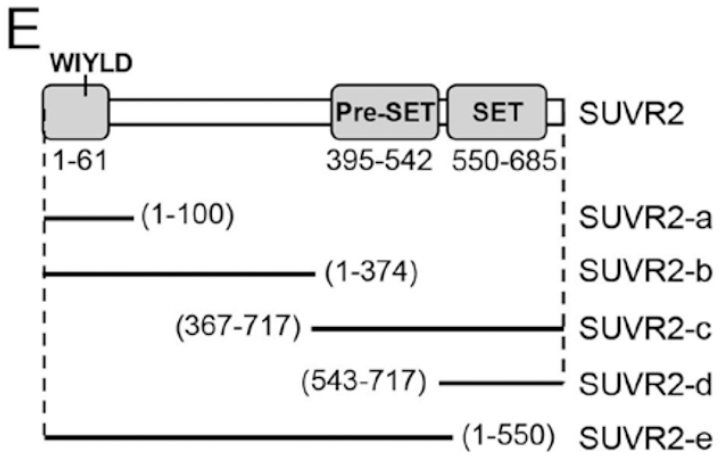

$\mathrm{F}$

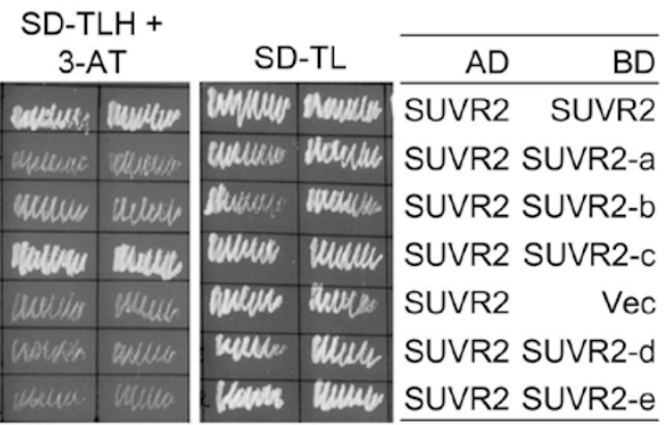

$440 \mathrm{KDa}$

$\downarrow$

$\mathrm{H}$

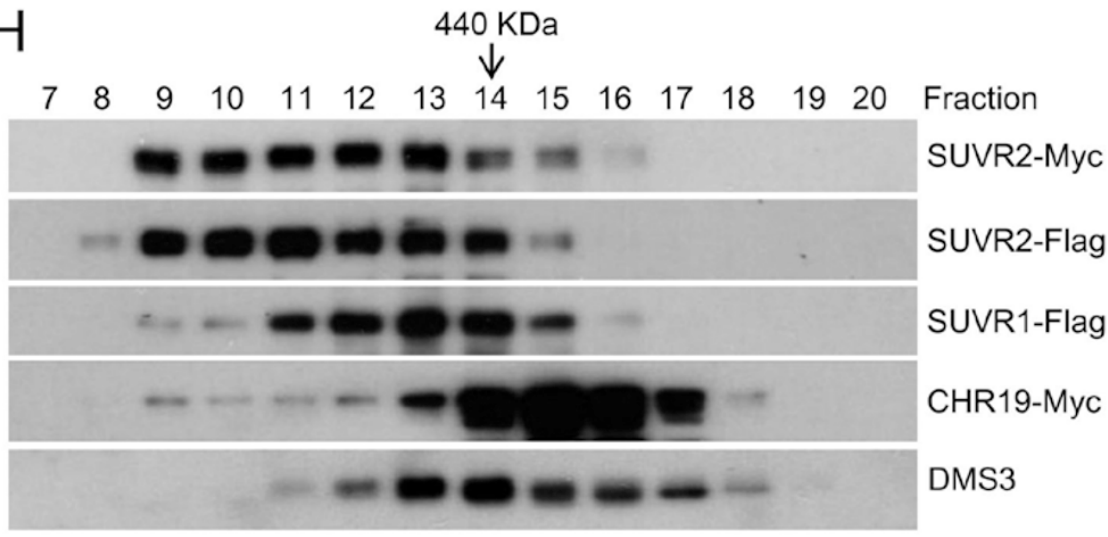

Figure 6 SUVR2 interacts with SUVR1 and the SNF2 chromatin-remodeling protein CHR19. (A) The interaction between SUVR1 and SUVR2 as determined by co-IP. The protein extract was isolated from the plants that express both SUVR1-Flag and SUVR2-Myc transgenes. The extract was immunoprecipitated by anti-Myc antibody and subjected to western blotting. (B) The interaction between SUVR2 and SUVR2 was determined by co-IP. The SUVR2-Myc transgene was combined with the SUVR2-Flag transgene in Arabidopsis. (C, D) GST pull-down assay was performed to test the interaction between GST-SUVR1 and HIS-SUVR2 (C) or HIS-SUVR1 (D). The GST protein was expressed and used as a control. (E) Diagrams of the fulllength and truncated SUVR2 protein sequences in the pGBKT7 vector. The constructs were used in yeast two-hybrid assay. (F) The yeast strains harboring the indicated constructs were grown on both SD-TL and SD-TLH (the synthetic dropout medium minus Trp, Leu, and His). $20 \mathrm{mM}$ 3-AT was added in SD-TLH to inhibit the growth of yeast strains. (G) The interaction of SUVR2 with CHR19 was determined by co-IP. The CHR19-Myc transgene was combined with the SUVR2-Flag transgene in Arabidopsis. (H) The elution profiles of SUVR2-Myc, SUVR2-Flag, SUVR1-Flag, CHR19-Myc, and DMS3 in gel filtration assay. Anti-Myc, anti-Flag antibody, and DMS3-specific antibody were used in western blotting.

1suvr2 double mutant (Figure 1C). We tested the effect of suvr2 on transcriptional silencing at several other previously identified RdDM target loci [47,65]. Our results indicated that AT1TE51360, AT2TE78930, ERT7, ERT12, and ERT14 but not ERT9 are derepressed in the suvr2 mutant (Figure 8B, Supplementary information, Figure S10A), further confirming that SUVR2 acts in transcriptional silencing at a subset of RdDM target loci. To 
determine the function of CHR19, CHR27, and CHR28 in transcriptional silencing, we obtained the mutants of CHR19, CHR27, and CHR28, which are Salk_054130C, Salk_063135C, and Salk_057016C, respectively, and generated a chr19chr27chr28 triple mutant (chr19/27/28) by crossing. No morphological phenotype was visualized in the chr19/27/28 triple mutant and each of the single mutants. Quantitative RT-PCR analysis indicated that the silencing of solo LTR and ERT14 is clearly affected in the chr19, chr27, and chr28 single mutants, and the effect is markedly enhanced in the chr19/27/28 triple mutant (Figure 8C, Supplementary information, Figure S10B). Thus, the function of CHR19, CHR27, and CHR28 in the silencing of solo LTR and ERT14 is at least partially redundant. The silencing of AT1TE51360, AT2TE78930, $E R T 7, E R T 9$, and ERT12 is either not affected or slightly affected in the chr19, chr27, and chrl8 single mutants but is significantly relieved in the chr19/27/28 triple mutant (Figure 8D, Supplementary information, Figure S10B). The function of CHR19, CHR28, and CHR29 in transcriptional silencing at these loci is clearly redundant. The silencing of $A t G P 1$ and $S D C$ is not markedly affected even in the chr19/27/28 triple mutant (Figure 8C), suggesting that CHR19/27/28 are not required for the silencing of $A t G P 1$ and $S D C$.

These results indicate that solo LTR, AT1TE51360, AT2TE78930, ERT7, ERT12, and ERT14 are not only targeted by SUVR2 but also by CHR19/27/28, suggesting that CHR19, CHR27, and CHR28 are functionally associated with SUVR2 at these loci. However, AtGP1 and SDC are targeted by SUVR2 but not by CHR19/27/28 (Figure $8 \mathrm{~A}$ and $8 \mathrm{C}$ ), whereas ERT9 is targeted by CHR19/27/28 but not by SUVR2 (Supplementary information, Figures S10A and S9B). These results suggest that SUVR2 and CHR19/27/28 function together at some target loci but have independent roles at other loci.

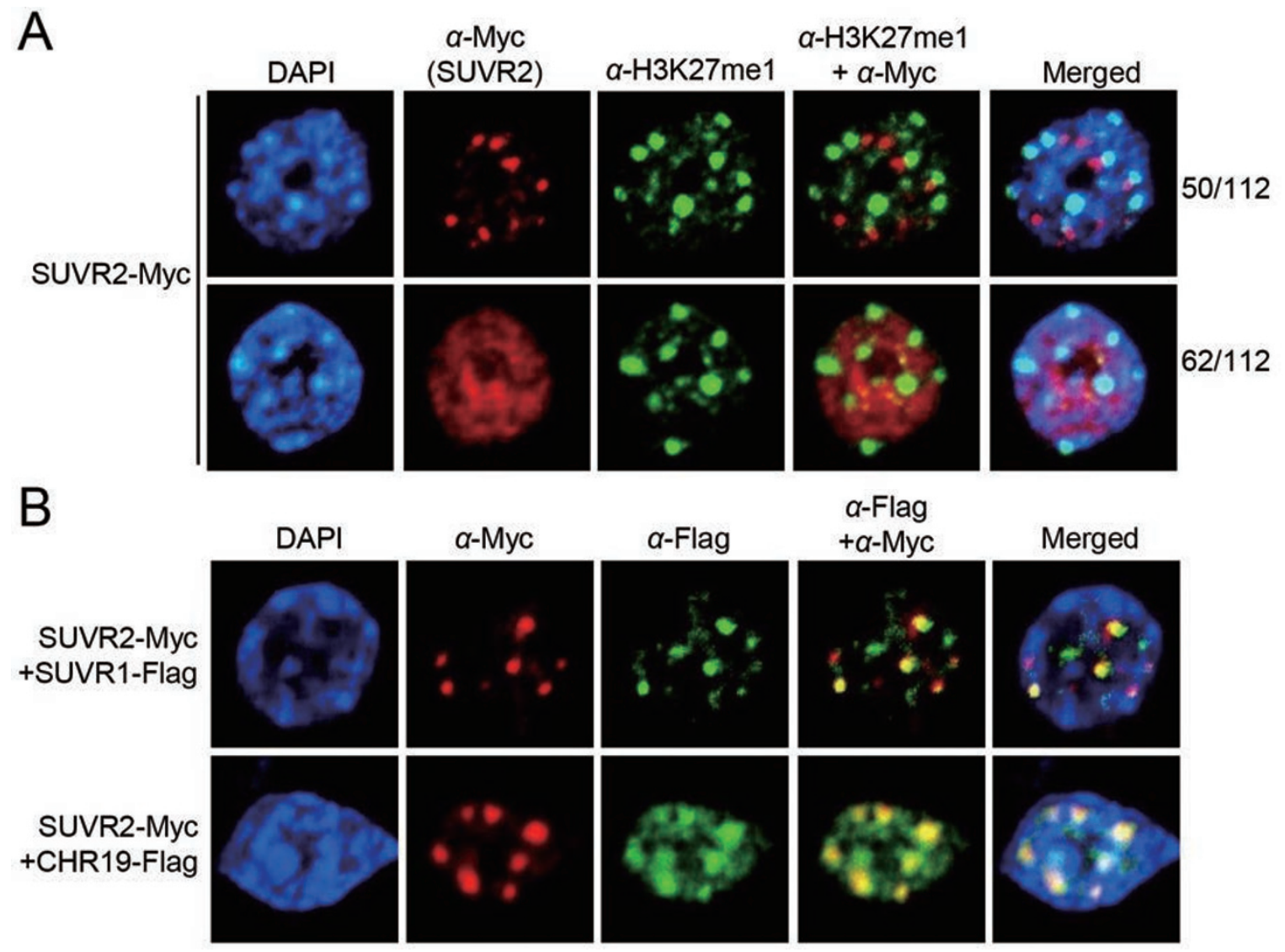

Figure 7 SUVR2 colocalizes with SUVR1 and CHR19 in the nucleus. (A) The SUVR2-Myc signals are shown in nuclei. The SUVR2-Myc signals were detected by anti-Myc antibody. Anti-H3K27me antibody was used for immunostaining to indicate heterochromatin foci. The nuclei were stained by DAPI as a control. (B) Coimmunolocalization was performed to determine whether SUVR2 is colocalized with SUVR1 and CHR19. SUVR1-Flag and CHR19-Flag constructs were separately introduced into the SUVR2-Myc transgenic plants. The nuclei were extracted from the offspring plants and subjected to coimmunostaining with both anti-Myc antibody and anti-Flag antibody. 
SUVR2 is required for the silencing of the RD29A$L U C$ transgene in the ros 1 mutant background (Figure $1 \mathrm{~A}$ and $1 \mathrm{~B})$. To determine whether CHR19 is required for the silencing of RD29A-LUC, we introduced the chr19 mutation into the ros 1 mutant by crossing. Luminescence imaging of ros 1 chrl 19 indicated that the chr 19 mutation relieves the silencing of RD29A-LUC in ros 1 (Figure $8 \mathrm{E}$ ), which was confirmed by quantitative RT-PCR (Figure $8 \mathrm{~F}$ ). These results suggest that like SUVR2, CHR19 is involved in the silencing of the RD29A-LUC transgene. The effect of chr19 on the silencing of RD29A$L U C$ is much weaker than that of suvr2 (Figure 8E and
8F), which is consistent with the observation that CHR19 is functionally redundant with $\mathrm{CHR} 27$ and CHR28 in the transcriptional gene silencing of many loci (Figure 8C and 8D, Supplementary information, Figure S10B).

The effect of suvrl on the silencing of RdDM target loci was determined by quantitative RT-PCR. The result indicated that suvrl affects transcriptional gene silencing at a subset of SUVR2 target loci (Figure $8 \mathrm{C}$ and $8 \mathrm{D}$, Supplementary information, Figure S10A and S10B). The involvement of SUVR1 in the silencing of SUVR2 target loci is consistent with the finding that SUVR1 and SUVR2 interact with each other and form a tight
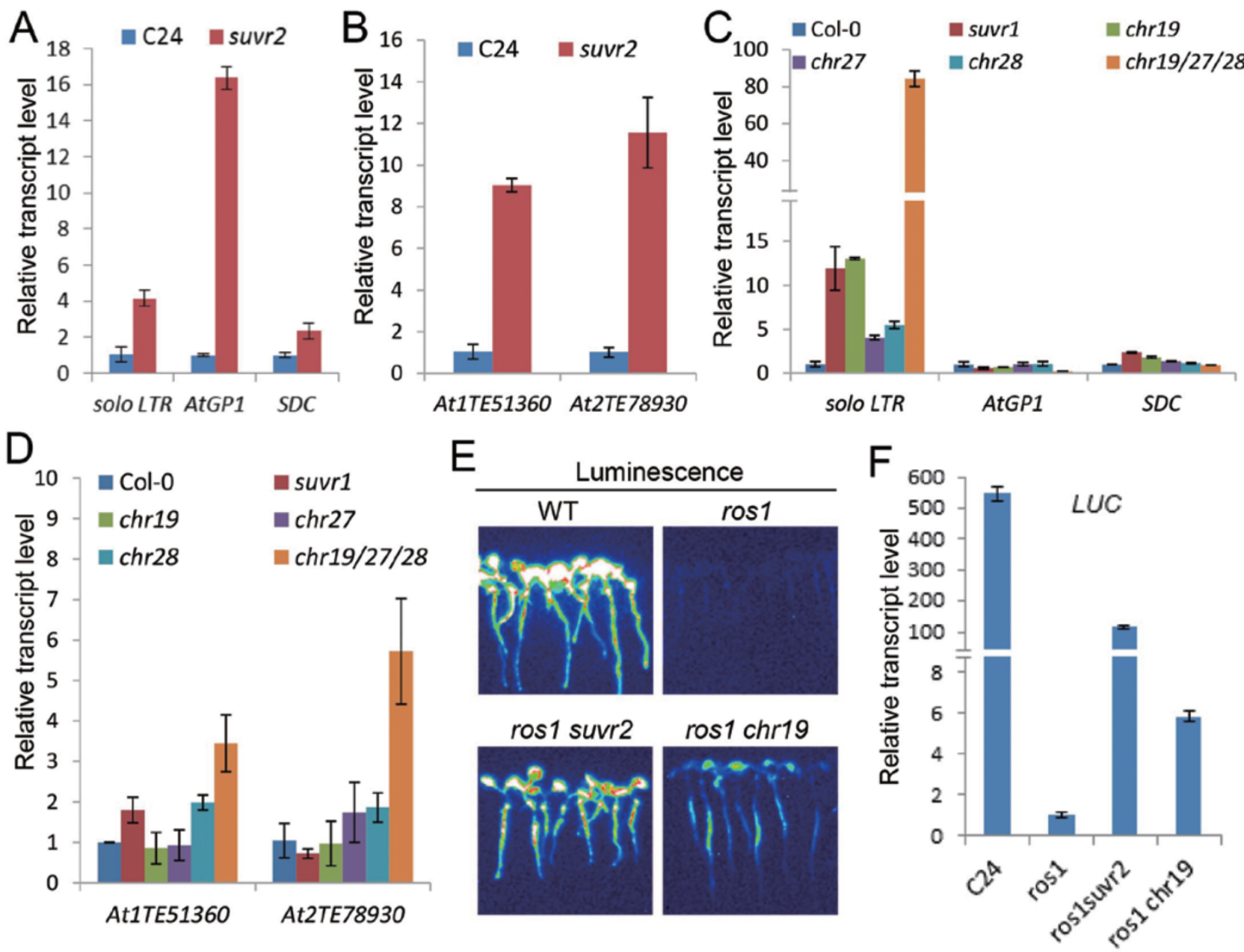

Figure 8 SUVR1 and the SNF2 chromatin-remodeling proteins CHR19, CHR27, and CHR28 are required for transcriptional gene silencing. (A) The effect of suvr2 on the RNA transcript levels of solo LTR, AtGP1, and SDC. The RNA transcript levels of these loci were determined by quantitative RT-PCR. (B) The effect of suvr2 on the RNA transcript levels of two transposable elements AT2TE51360 and AT2TE78930. (C) The effect of suvr1, chr19, chr27, and chr28 on the RNA transcript levels of solo LTR, AtGP1, and SDC. (D) The effect of suvr1, chr19, chr27, and chr28 on the RNA transcript levels of AT2TE51360 and AT2TE78930. (E) The effect of chr19 on the silencing of the RD29A-LUC transgene. The chr19 mutation was introduced into the ros1 mutant harboring RD29A-LUC transgene by crossing. Luminescence imaging of the wild-type plants, and the ros1, ros1suvr2, and ros1chr19 mutant plants is shown. (F) The expression of the luciferase reporter gene was determined by quantitative RT-PCR in the wild type, ros1, ros1suvr2, and ros1chr19 plants harboring the RD29A-LUC transgene. 
complex in vivo. To determine whether SUVR1 is required for transgene silencing, we introduced the suvr 1 mutation into the ros 1 mutant harboring the RD29A$L U C$ transgene. The suvr 1 mutation weakly suppresses the silencing of RD29A-LUC, and the effect of suvrl on transgene silencing is much weaker than that of suvr 2 (Supplementary information, Figure S11). We integrated the suvr 1 mutation into the ros 1 suvr 2 double mutant and found that in the roslsuvrlsuvr2 mutant, the expression of RD29A-LUC is not further enhanced compared to that in the ros 1suvr2 mutant (Supplementary information, Figure S11). These results suggest that SUVR1 and SUVR2 have non-redundant function and both of them are necessary for transcriptional gene silencing.

The function of SUVR2 and CHR19/27/28 in DNA methylation, siRNA accumulation, and nucleosome positioning

The bisulfite sequencing results showed that SUVR2 weakly affects DNA methylation at a subset of RdDM target loci (Figure 2B). Because CHR19, CHR27, and CHR28 associate with SUVR2 and are required for transcriptional gene silencing, we asked whether the function of CHR19, CHR27, and CHR28 in transcriptional gene silencing is related to DNA methylation. The transcript levels of solo LTR and ERT14 are increased in suvr2 and the chr19/27/28 triple mutant (Figure 8A and 8C, Supplementary information, Figure S10A and S10B). In the suvr2 mutant, consistent with the increased expression of solo LTR and ERT14, the DNA methylation levels at the two loci are weakly reduced (Figure 9A). In the chr19/27/28 mutant, DNA methylation is reduced for ERT14 but not for solo LTR even though the transcript levels of both ERT14 and solo LTR are increased (Figures 9A and 8C, Supplementary information, Figure S10B). Moreover, we found that the DNA methylation of the RdDM target locus IGN5 is not affected by suvr 2 and chr19/27/28, whereas the DNA methylation of another RdDM target locus $I G N 23$ is mildly reduced by suvr 2 but not by chr19/27/28 (Figure 9A). The aforementioned bisulfite sequencing assay indicated that suvr2 significantly reduces DNA methylation at solo LTR and $M E A-I S R$ sites (Figure 2B). We performed bisulfite sequencing to determine the effect of $c h r 19 / 27 / 28$ on DNA methylation at these two sites and found that their DNA methylation levels are not significantly affected in the chr19/27/28 mutant (Supplementary information, Figure $\mathrm{S} 12 \mathrm{~A}$ and $\mathrm{S} 12 \mathrm{~B}) .5 \mathrm{~S} r \mathrm{DNA}$ methylation was reduced by suvr2 at $\mathrm{CHH}$ sites and the effect of suvr 2 is weaker than that of nrpe1 (Supplementary information, Figure S12C). 5S rDNA methylation is slightly reduced by $c h r 19 / 27 / 28$ at $\mathrm{CHH}$ sites but the effect is even weaker than that of suvr2 (Supplementary information, Figure S12C). These results suggest that CHR19/27/28 affect DNA methylation to a lesser extent than SUVR2 and may have DNA methylation-independent roles in transcriptional gene silencing.

Previous reports suggest that RdDM components are required for $\mathrm{H} 3 \mathrm{~K} 9$ dimethylation at $\mathrm{RdDM}$ target loci $[11,57,58]$. We performed ChIP-PCR to test whether CHR19/27/28 are required for H3K9 dimethylation (Figure 9B). Our result indicated that the H3K9me2 levels at solo LTR and IGN5 are drastically decreased in the Pol V mutant nrpel (Figure 9B), which is consistent with previous reports $[11,57]$. However, the $\mathrm{H} 3 \mathrm{~K} 9 \mathrm{me} 2 \mathrm{ChIP}$ assay indicated that SUVR2 is not required for $\mathrm{H} 3 \mathrm{~K} 9 \mathrm{me} 2$ at its target loci including the transgenic and endogenous RD29A promoters, solo LTR, and IGN5 (Figure 5A-5D). Here, we found that CHR $19 / 27 / 28$ is dispensable for H3K9me2 at solo LTR and IGN5 (Figure 9B), which is consistent with the action of SUVR2 at these loci. Thus, the involvement of SUVR2 and CHR19/28/29 in transcriptional silencing is mostly likely through an as yet unknown mechanism.

The function of SUVR2 and CHR19/27/28 in small RNA accumulation was examined by northern blotting (Figure 9C). The accumulation of Pol IV- and Pol V-dependent siRNAs including AtREP2 siRNA, solo $L T R$ siRNA, and siRNA1003 is slightly decreased in suvr2 (Figure 9C). The accumulation of miRNA171 and trans-acting siRNA255 is not affected by suvr2 (Figure 9C), suggesting that the function of SUVR2 is specifically associated with Pol IV-dependent siRNAs but not with other types of small RNAs. However, the effect of suvr2 on siRNA accumulation is much weaker than that of the Pol V mutation nrpel (Figure 9C). The effect of suvr2 on Pol IV- and Pol V-dependent siRNAs as determined by northern blotting is consistent with our small RNA deep sequencing data (Figure $2 \mathrm{C}$ and 2D, Supplementary information, Table S1). In the chr19/27/28 mutant, the accumulation of AtREP2 siRNA, solo LTR siRNA, and siRNA1003 is weakly decreased (Figure 9C). The effect of chr19/27/28 on siRNA accumulation is similar to that of suvr2. Moreover, neither suvr2 nor chr19/27/28 affects the accumulation of siRNA02, which shows a Pol IV-dependent and Pol V-independent pattern (Figure 9C). Thus, suvr 2 and chr19/27/28 may indirectly affect the accumulation of Pol IV- and Pol V-dependent siRNAs at RdDM target loci.

As CHR19/27/28 are putative ATP-dependent SNF2 chromatin-remodeling proteins, it is possible that the involvement of CHR 19/27/28 in transcriptional gene silencing is correlated with their function in nucleosome positioning. Pol V-stabilized nucleosomes were previous- 
A

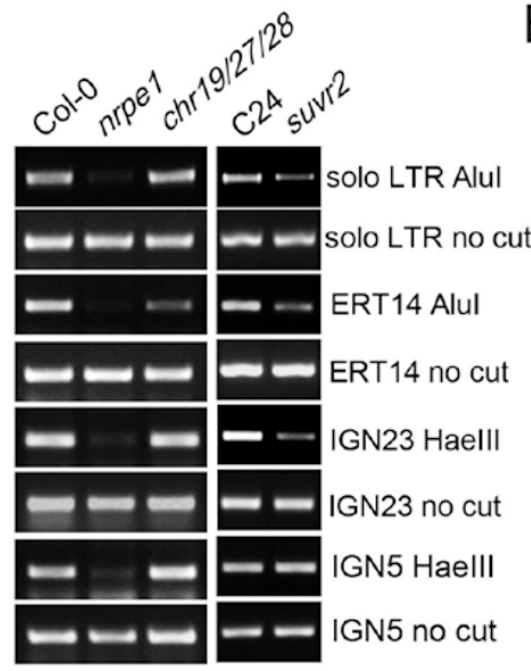

B

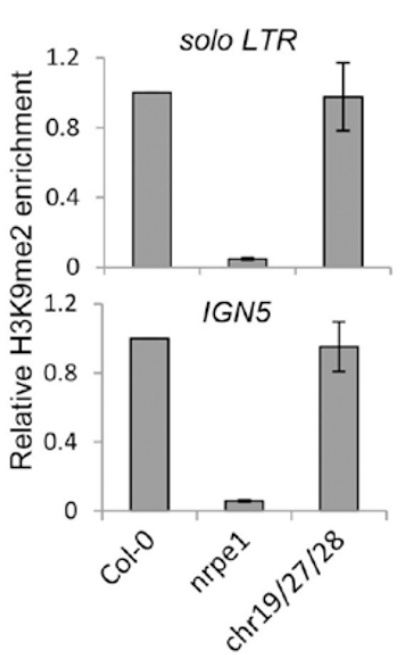

C

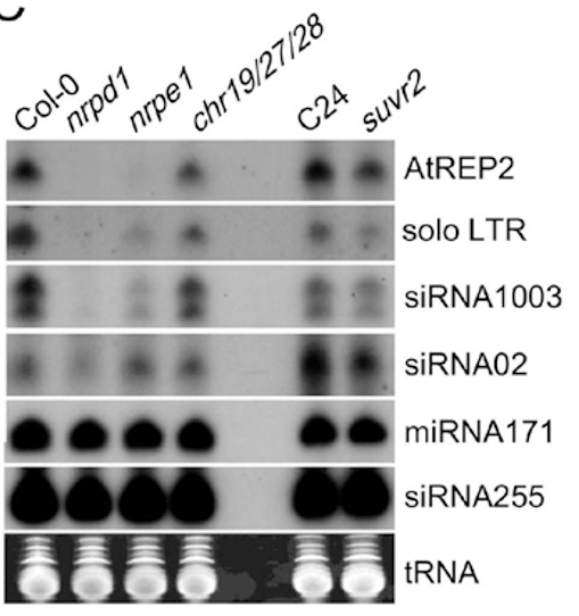

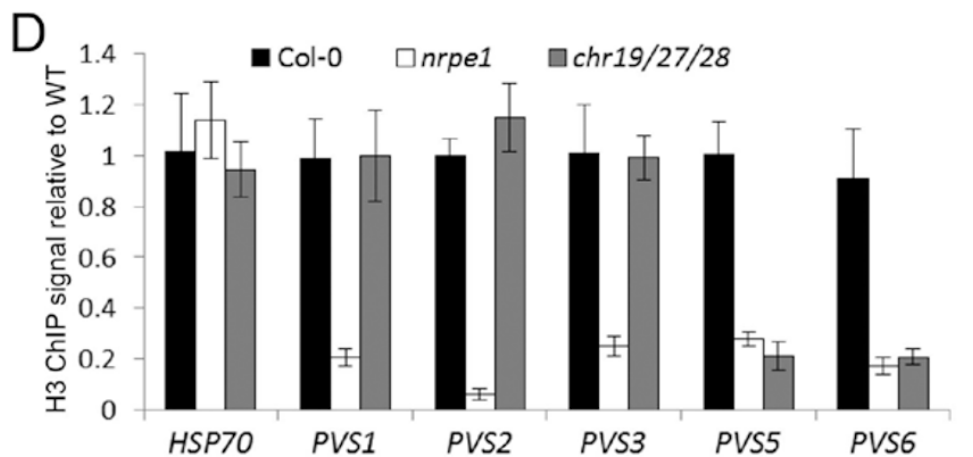

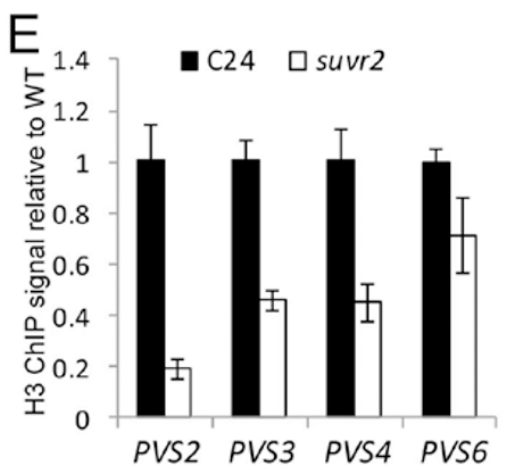

Figure 9 SUVR2 and the SNF2 chromatin-remodeling proteins are involved in DNA methylation, siRNA accumulation, and nucleosome positioning. (A) The effect of suvr2 and chr19/27/28 on DNA methylation at RdDM target loci. Genomic DNA was digested by the DNA methylation-sensitive restriction enzyme Alul or Haelll, followed by PCR. (B) The effect of $n r p e 1$ and chr19/27/28 on H3K9 dimethylation at RdDM target loci solo LTR and IGN5. (C) The effect of suvr2 and chr19/27/28 on the accumulation of Pol IV-dependent siRNAs. Small RNA accumulation was measured by small RNA northern blotting. miRNA171 and ta-siRNA255 were included as controls that are not affected in the Pol IV mutant nrpd1. The tRNA image on the ethium bromide-stained gel was shown as a small RNA loading control. (D, E) The effect of nrpe1, chr19/27/28, and suvr2 on Pol V-stabilized nucleosome positioning. The occupancy of nucleosomes on chromatin was determined by MNase digestion followed by H3 ChIP and quantitative PCR. The ChIP signal of HSP70 was shown as a negative control [47]. ChIP signals were normalized to the actin gene ACT2.

ly identified by MNase (Microccocal Nuclease) digestion followed by DNA deep sequencing [47]. In the Pol V mutant nrpe1, these nucleosomes were destabilized and their levels decreased [47]. We performed MNase digestion followed by H3 ChIP-PCR to determine whether chr19/27/28 may affect the occupancy of these nucleosomes on chromatin (Figure 9D). The nucleosome occupancy at PVS1, PVS2, PVS3, PVS5, and PVS6 sites was markedly decreased in nrpel (Figure 9D), which is consistent with the previous report [47]. In chr19/27/28, the nucleosome occupancy was reduced at the PVS5 and PVS6 sites but not at the PVS1, PVS2, and PVS3 sites
(Figure 9D), suggesting that CHR19/27/28 are required for the occupancy of a subset of Pol V-stabilized nucleosomes on chromatin. The suvr2 mutant used in this study is in the C24 ecotype, in which the occupancy of Pol V-stabilized nucleosomes is detectable at PVS2, PVS3, PVS4, and PVS6 (Figure 9E). In the suvr2 mutant, the occupancy of Pol V-stabilized nucleosomes is reduced at all these loci (Figure 9E). These results are consistent with the finding that CHR19/27/28 are required for transcriptional gene silencing at a subset of SUVR2 target loci (Figure 8A-8D). Based on a previous report [47], the SWI/SNF chromatin-remodeling complex component 
Brahma (BRM) affects the same subset of the nucleosome sites, suggesting that CHR19/27/28 and BRM may be recruited to specific chromatin target loci by the same mechanism. Pol V-produced noncoding RNAs are thought to recruit IDN2 and then the SWI/SNF complex to chromatin, thereby facilitating nucleosome positioning [47]. Pol V-produced noncoding RNAs are probably not only required for the recruitment of the SWI/SNF complex but also for the recruitment of CHR19/27/28.

\section{Discussion}

SUVR2 is a member of the SU(VAR)3-9-related protein family in Arabidopsis. In this family, SUVR4 is an active histone methyltransferase and is involved in histone $\mathrm{H} 3 \mathrm{~K} 9$ methylation and transcriptional gene silencing [28], but the histone methyltransferase activity of SUVR4 is much weaker than that of the human SUV39H1 as determined by an in vitro assay [28]. SUVR1, SUVR2, and SUVR5 have no histone methyltransferase activity as determined by in vitro assays (Figure $5 \mathrm{G}$ ) $[28,30]$, even though SUVR5 is involved in histone H3K9 methylation in vivo $[28,30]$. The SET domain of SUVR2 is conserved in the SU(VAR)3-9 homologs including Dim-5, Clr4, and G9a (Supplementary information, Figure S6B). Previous structural and biochemical studies suggest that some conserved residues in the SET domains of the SU(VAR)3-9 homologs are directly responsible for the binding of the substrate histone $\mathrm{H} 3$ and the methyl group donor SAM [20, 64]. These residues are critical for the catalytic activity of the histone methyltransferases. In the SET domain of SUVR2, substitutions are present in some of these critical residues, which include the H3K9-binding sites corresponding to N247 and F281 of Dim-5, as well as the SAM-binding sites corresponding to R155 and Y204 of Dim-5 (Supplementary information, Figure S6B). These substitutions are likely responsible for the loss of the SUVR2 histone methyltransferase activity. Our study here showed that mutations of the conserved catalytic residues in the SET domain of SUVR2 do not affect the function of SUVR2 in transcriptional gene silencing (Figure 5E-5F), which is consistent with the observation that SUVR2 has no histone methyltransferase activity as determined by the in vitro assay. In Arabidopsis, the three SU(VAR)3-9 homologs SUVH4/KYP, SUVH5, and SUVH6 have histone $\mathrm{H} 3 \mathrm{~K} 9$ methyltransferase activity as determined by in vitro and in vivo assays $[21,22,25]$. The three active H3K9 methyltransferases contain a conserved SRA domain that can directly bind to both symmetric and asymmetric cytosine sites [24, 25]. Histone H3K9 dimethylation at RdDM target loci is likely catalyzed by SUVH4/
KYP, SUVH5, and SUVH6 in Arabidopsis.

The WILYD domain was previously identified as the domain required for ubiquitin binding in SUVR4 [29]. The domain is thought to be responsible for conversion of the enzyme activity of SUVR4 from H3K9 dimethyltransferase to trimethyltransferase. Unlike SUVR4, no histone methyltransferase activity was detected for SUVR2 even when ubiquitin was added (Figure 5G), suggesting that the WILYD domain of SUVR2 may have a different role compared to that of SUVR4. We found that mutations of the conserved residues in the WILYD domain do not affect the function of SUVR2 in transcriptional gene silencing. Thus, the ubiquitin-binding ability of the WILYD domain is dispensable for the function of SUVR2 in transcriptional gene silencing.

SUVR2 was recently reported as a canonical RdDM component [5]. We found that DNA methylation and transcriptional silencing is affected by suvr 2 at a subset of RdDM target loci, which is consistent with a role of SUVR2 in the RdDM pathway. Although suvr2 significantly affects transcriptional gene silencing at RdDM target loci, DNA methylation is either not affected or weakly affected in the suvr2 mutant (Figure 2A and 2B). These results suggest that SUVR2 may also function downstream of the RdDM pathway or may have an RdDM-independent role in transcriptional gene silencing.

Our observation that suvr2 shows synergistic effects with canonical RdDM mutations on the silencing of a subset of RdDM target loci (Figure 3B) supports that SUVR2 functions in transcriptional gene silencing at least partially independently of RdDM. Our results suggest that SUVR2 functions not only at a subset of RdDM target loci but also at loci that are not targeted by RdDM (Figure 4A-4F, Supplementary information, Tables S3S6). Based on our quantitative RT-PCR results (Figure 3B), common target loci shared by SUVR2 and RdDM can be divided into two classes. Class I loci are represented by solo LTR and AtGP1. These loci are not further activated by suvr 2 when the RdDM pathway is completely disrupted in the nrpdl and nrpel mutant backgrounds. The existence of class I loci suggests that SUVR2 and RdDM act non-redundantly in transcriptional gene silencing. At these loci, SUVR2 probably acts downstream of the RdDM pathway and is required for the transduction of DNA methylation to transcriptional repression. Class II loci are represented by SDC and ERT7 (Figure 3B). The transcription of class II loci is synergistically activated when suvr2 is combined with nrpdl or nrpel. The synergistic effect between suvr 2 and canonical RdDM mutations indicates that the function of SUVR2 is independent of the RdDM pathway at class II loci. These 
results suggest that the cooperation between SUVR2 and $\mathrm{RdDM}$ is more complex than expected. Some specific chromatin features in class I and class II loci may determine how SUVR2 cooperates with RdDM.

Previous reports indicate that the RdDM pathway cooperates with several other chromatin silencing regulators including DDM1, HAD6, MOM1, and MORC6/ DMS11 [26, 34, 39, 41, 42, 65]. These results suggest that a stable state of chromatin silencing is dependent on the interplay of multiple transcriptional silencing mechanisms. It is interesting to understand when and how SUVR2 mediates transcriptional gene silencing in DNA methylation-dependent and -independent manners. The association of SUVR2 with the chromatin-remodeling proteins CHR19, CHR27, and CHR28 provides a possible functional mechanism for SUVR2 in transcriptional gene silencing. Several chromatin-remodeling proteins were previously identified as regulators of transcriptional gene silencing [31-35]. The chromatin-remodeling proteins DRD1 and CLSY1 act as RdDM regulators [32, 33]. DRD1 interacts with DMS3 and RDM1, forming a DDR complex required for the occupancy of Pol V on chromatin and for Pol V transcription $[11,15,16]$. CLSY1 is required for proper localization of NRPD1 and RDR2 in the nucleus [33]. DDM1 facilitates the access of DNA methyltransferases to H1-containing heterochromatin and is required for maintenance of DNA methylation [34]. These results suggest that chromatin remodeling mediated by DRD1, CLSY1, and DDM1 contributes to DNA methylation and transcriptional gene silencing. We demonstrate that the involvement of SUVR2 and CHR19/27/28 in transcriptional gene silencing is not always correlated with DNA methylation. Pol V-stabilized nucleosomes were thought to participate in transcriptional gene silencing [47]. We found that SUVR2 is required for Pol V-stabilized nucleosome positioning on chromatin, whereas CHR19/27/28 are responsible for the occupancy of a subset of these nucleosomes (Figure 9D and 9E). Previous studies suggest that nucleosome positioning is related to both DNA methylation and Pol II transcription $[66,67]$. The involvement of SUVR2 and CHR19/27/28 in nucleosome positioning provides a possible DNA methylation-independent mechanism underlying transcriptional gene silencing.

SUVR2 is required for nucleosome positioning at all tested Pol V-stabilized nucleosome loci, whereas CHR19/27/28 are only responsible for nucleosome positioning at a subset of these loci (Figure 9D and 9E). A previous report indicated that a SWI/SNF chromatin-remodeling complex is required for nucleosome positioning for a subset of Pol V-stabilized nucleosome loci [47]. We found that CHR19/27/28 are involved in nucleosome positioning at the same subset of Pol V-stabilized nucleosomes as the SWI/SNF complex (Figure 9D). The recruitment of the SWI/SNF complex to chromatin was thought to be guided by Pol V-produced noncoding RNAs and IDN2 [47]. CHR19/27/CHR28 may be recruited to chromatin in a similar manner to the SWI/SNF complex and are involved in nucleosome positioning at a subset of RdDM target loci. We demonstrated that SUVR2 directly binds to histone $\mathrm{H} 3$ in vitro (Figure $5 \mathrm{H}$ ). The binding of SUVR2 to histone H3 may be necessary for the interaction of SUVR2 to chromatin. Thus, in addition to Pol V-produced nocoding RNAs and IDN2, SUVR2 is probably required for the recruitment of CHR19/27/28 to chromatin. The recruited chromatin-remodeling proteins then mediate nucleosome positioning, thereby contributing to DNA methylation or directly repressing Pol II transcription.

Our results demonstrated that SUVR2 and CHR19/27/28 weakly affect the accumulation of Pol IV- and Pol V-dependent 24-nt siRNAs (Figures 2C, 2D and 9C). Pol V is responsible for producing scaffold noncoding RNAs and acts downstream of siRNA biogenesis [11]. However, in the RdDM pathway, Pol V is also involved in accumulation of a subset of Pol IV-dependent siRNAs [68]. The effect of Pol V on siRNA accumulation was thought to depend on its function in DNA methylation and transcriptional gene silencing [68, 69]. Similarly, SUVR2 and CHR19/27/28 may affect siRNA accumulation through a self-reinforcing loop between siRNA accumulation and transcriptional gene silencing at RdDM target loci.

The function of SUVR2 in transcriptional gene silencing is not always dependent on the chromatin-remodeling proteins CHR19/27/28 (Figure 8A-8D). Although SUVR2 associates with CHR19/27/28, the size of the main SUVR2 complex is much higher than that of the main CHR19 complex (Figure 6H), suggesting that SUVR2 forms a separate complex without CHR19. The fractions of the SUVR2 complex largely overlap with those of the SUVR1 complex (Figure 6H). We propose that SUVR2 not only associates with CHR19/27/28 but also forms a heteromer with SUVR1, thereby facilitating DNA methylation and transcriptional gene silencing. Further studies are required to clarify how the SUVR1/ SUVR2 heteromer participates in transcriptional gene silencing in a CHR19/27/28-independent manner.

\section{Materials and Methods}

Plant materials, map-based cloning, and complementation
testing
$\quad$ Arabidopsis seedlings were grown on MS medium for ten 
days and were then subject to luminescence imaging after cold treatment at $4{ }^{\circ} \mathrm{C}$ for 4 days. For kanamycin resistance detection, seedlings were grown on $150 \mathrm{mM}$ kanamycin MS medium and were imaged after two weeks. For map-based cloning, the ros $1 \# 87$ double mutant in the $\mathrm{C} 24$ ecotype was crossed to the T-DNA insertion line of the ros 1 mutant in the Col-0 ecotype (Salk_045303), and the F2 generation was used for mapping. The bright ones of F2 were selected for DNA extraction followed by PCR of SSLP (Simple sequence length polymorphism) markers. The mutation was mapped to a $974 \mathrm{~kb}$ interval on the chromosome 5. The ros $1 \# 87$ genome was sequenced, and a G-to-A mutation was found in SUVR2 at the junction of the second intron and the third exon. The full genomic sequence of SUVR2 with its own promoter was cloned into the vector modified from pCAMBIA1305 to have a $3 \times$ Flag tag at its 3 '-end. This construct was transformed into ros $1 \# 87$ for complementation assay. For the point mutation rescue test, the genomic sequence of SUVR2 with each indicated mutation was cloned and transformed into ros $1 \# 87$ as well. The ros 1 suvr 2 double mutant was crossed with the wild-type and the suvrl mutant (Salk_012786C) to obtain suvr2, ros1suvr1, and ros1suvr1suvr2. Moreover, we crossed ros 1suvr2 with ros 1nrpd1, ros1nrpe1, and ros $1 d \mathrm{dcl} 3$ to obtain ros1nrpd1suvr2, ros 1nrpe1suvr2, and ros $1 d \mathrm{dcl}$ 3 suvr2, respectively. The chr19, chr27, and chr28 single mutants were used to generate the $c h r 19 \operatorname{chr} 27 \mathrm{chr} 28$ triple mutant by crossing. The chr19 mutant was crossed with the ros 1 mutant harboring the RD29A-LUC transgene to obtain the ros 1chr19 double mutant.

\section{DNA methylation assay}

DNA methylation was determined by bisulfite sequencing, Southern blotting, and chop-PCR. For bisulfite sequencing, genomic DNA from 2-week-old seedlings was extracted and $2 \mu \mathrm{g}$ of genomic DNA was treated by the sodium bisulfite reagent (Qiagen, 59104) so that unmethylated cytosines were converted to uracils. The converted DNA was purified and used for PCR with primers indicated in Supplementary information, Table S8. The PCR products were cloned into T-vector for sequencing. For each sample, more than 15 clones were sequenced, and the percentage of $\mathrm{CG}$, $\mathrm{CHG}$ and $\mathrm{CHH}$ methylation was analyzed separately online by CyMate website [70]. For Southern blotting, $5 \mu \mathrm{g}$ of genomic DNA was digested with the DNA methylation-sensitive restriction enzymes HpaII, MspI, and HaeIII. The digested DNA was subjected to Southern blotting for $5 S r D N A$. For chop-PCR, genomic DNA was digested with the DNA methylation sensitive restriction enzymes HaeIII and AluI overnight, followed by amplification of ERT14, solo LTR, IGN5, and IGN23.

\section{RNA analysis and small RNA northern blotting}

For quantitative RT-PCR, total RNA was extracted using Trizol reagent, followed by reverse transcription and PCR (TaKaRa, RR012A). For the detection of Pol V-produced transcripts, onestep RT-PCR was performed as described previously [11]. For RNA-seq analysis, total RNA was isolated from two-week-old seedlings and used for generating RNA libraries. The RNA libraries were subjected to single-end sequencing by HiSeq 2000 (Illumina). RNA-seq data were analyzed as described before [71].

For small RNA northern blotting, small RNA was extracted from two-week-old seedlings with Trizol reagent, and run on a $15 \%$ polyacrylamide gel with the method described [49]. The small RNAs were transferred to Hybond $\mathrm{N}^{+}$membrane electrically
(Amersham, RPN3050N), and then subjected to small RNA hybridization. Two types of probes were used: DNA oligonucleotides or PCR products. DNA oligonucleotides were labeled with $\gamma-{ }^{32} \mathrm{P}-$ ATP, and PCR products with $\alpha-{ }^{32} \mathrm{P}-\mathrm{dCTP}$. The membrane was incubated in PerfectHyb buffer (Sigma, H7033) overnight at $38^{\circ} \mathrm{C}$ for hybridization.

\section{Affinity purification and mass spectrometry}

Six grams of flower tissue from the SUVR2-Flag transgenic plants as well as the wild-type control were used to prepare protein extracts as previously described [8]. Anti-Flag M1 agarose (Sigma, A4596) was incubated with the protein extracts and washed. The agarose-bound proteins were eluted with $3 \times$ Flag peptides (Sigma) and run on SDS-PAGE followed by silver staining (Sigma, PROTSIL1). The silver-stained proteins were de-stained and digested ingel with trypsin $(10 \mathrm{ng} / \mu \mathrm{l}$ trypsin, $50 \mathrm{mM}$ ammonium bicarbonate, $\mathrm{pH}$ 8.0) at $37{ }^{\circ} \mathrm{C}$ overnight. The digested peptides were purified for mass spectrometry as previously reported [44].

\section{Immunolocalization}

Nuclei extracted from rosette leaves were fixed in $4 \%$ formaldehyde and then applied to slides as previously described [8]. The slides were blocked in PBS buffer with 3\% BSA, followed by primary antibody hybridization. Secondary anti-mouse antibody TRITC (Tetramethyl Rhodamine Isothiocyanate-conjugated) (Invitrogen, Z25005) and anti-rabbit antibody FITC (Fluorescein Isothiocyanate-conjugated) (Invitrogen, 710369) were added after washing of slides, and incubated on slides at $37{ }^{\circ} \mathrm{C}$. Chromatin was counterstained with DAPI (4'-6-Diamidino-2-phenylindole). Images were collected by SPINNING DISK confocal microscopy. The antibodies for H3K9me1, H3K9me2, H3K9me3, H3K27me1, $\mathrm{H} 3 \mathrm{~K} 27 \mathrm{me}$, and H3K27me3 were from Millipore.

\section{Coimmunoprecipitation}

SUVR2-Flag and SUVR2-Myc were constructed in the modified pCAMBIA1305 vector and introduced into Arabidopsis. $S U V R 2-M y c$ transgenic plants were crossed to the plants harboring SUVR1-Flag, SUVR2-Flag, NRPE1-Flag, KTF1-Flag, or DRM2Flag transgene, whereas SUVR2-Flag transgenic plants were crossed to the CHR19-Myc or RDM1-Myc transgenic plants to generate the offspring plants harboring two tagged proteins. Protein extracts were isolated from the plants and incubated with antic-Myc agarose (Sigma, A7470), anti-Flag M1 agarose (Sigma, A 4596), and anti-AGO4 conjugated agarose (Agrisera, AS09617). After the agarose-bound proteins were washed, the proteins were boiled and run on SDS-PAGE for western blotting.

\section{Histone methyltransferase activity assay}

The full-length SUVR2 and the SET domain of SUVR2 were cloned in frame with $6 \times$ His in the pET28a vector, and expressed in bacteria. The histone methyltransferase activity assay was carried out according to the method previously described [29]. The human SU(VAR)3-9 homolog G9a was served as a positive control.

\section{Chromatin immunoprecipitation}

$\mathrm{H} 3 \mathrm{~K} 9 \mathrm{me} 2$ and $\mathrm{H} 3 \mathrm{~K} 9 \mathrm{me} 3 \mathrm{ChIP}$ assays were carried out following the procedure previously described [11]. Two-week-old seedlings were fixed in $1 \%$ formaldehyde followed by washing for 5 
times. Nuclei were extracted from the material and incubated with H3K9me2 antibody (abcam, ab1220) or H3K9me3 antibody (Millipore, 17-625) at $4{ }^{\circ} \mathrm{C}$ overnight. Chromatin bound by H3K9me2 or H3K9me3 was purified and used for PCR with sequence-specific primers listed in Supplementary information, Table S8. The occupancy of $\mathrm{H} 3 \mathrm{~K} 9 \mathrm{me} 2$ or $\mathrm{H} 3 \mathrm{~K} 9 \mathrm{me} 3$ on the actin gene $A C T 2$ was used as a negative control. We determined nucleosome positioning according to the method described previously [47]. Briefly, the nuclear extract was digested with microccocal nuclease (MNase, NEB, \#M0247S) followed by H3 ChIP (abcam, ab1791) and quantitative PCR.

\section{Yeast two-hybrid assay}

The interaction of SUVR2 with either the full-length or truncated SUVR2 was determined by yeast two-hybrid assay. The fulllength and truncated versions of SUVR2 were constructed in the pGBKT7 plasmid, whereas the full-length SUVR2 was constructed in the pGADT7 plasmid. The pGBKT7 and pGADT7 plasmids were cotransformed into the yeast strain. The transformed yeast cells were grown on SD-TL (the synthetic dropout medium minus Trp and Leu) and the positive colonies were used for yeast two-hybrid. All the yeast strains harboring pGADT7 and pGBKT7 constructs were grown on SD-TLH (the medium minus Trp, Leu, and His) supplemented with $20 \mathrm{mM} 3$-AT as well as on SDTL. The growth on SD-TLH indicates the expression of the His synthesis gene, suggesting the interaction of the proteins in the pGADT7 and pGBKT7 plasmids.

\section{Acknowledgments}

We thank Drs Niu Huang and Wei Li for analyzing the putative SUVR2 structure. This work was supported by the National Basic Research Program of China (973 Program; 2012CB910900 and 2011CB812600) to X-J H, and by National Institutes of Health Grants (R01GM070795 and R01GM059138) to J-K Z.

\section{References}

1 Slotkin RK, Martienssen R. Transposable elements and the epigenetic regulation of the genome. Nat Rev Genet 2007; 8:272-285.

2 Matzke MA, Mosher RA. RNA-directed DNA methylation: an epigenetic pathway of increasing complexity. Nat Rev Genet 2014; 15:394-408.

3 Law JA, Jacobsen SE. Establishing, maintaining and modifying DNA methylation patterns in plants and animals. Nat Rev Genet 2010; 11:204-220.

4 He XJ, Chen T, Zhu JK. Regulation and function of DNA methylation in plants and animals. Cell Res 2011; 21:442465.

5 Stroud H, Greenberg MV, Feng S, Bernatavichute YV, Jacobsen SE. Comprehensive analysis of silencing mutants reveals complex regulation of the Arabidopsis methylome. Cell 2013; 152:352-364.

6 Haag JR, Pikaard CS. Multisubunit RNA polymerases IV and $\mathrm{V}$ : purveyors of non-coding RNA for plant gene silencing. Nat Rev Mol Cell Biol 2011; 12:483-492.

7 Qi Y, He X, Wang XJ, Kohany O, Jurka J, Hannon GJ. Dis- tinct catalytic and non-catalytic roles of ARGONAUTE4 in RNA-directed DNA methylation. Nature 2006; 443:10081012.

8 Pontes O, Li CF, Costa Nunes P, et al. The Arabidopsis chromatin-modifying nuclear siRNA pathway involves a nucleolar RNA processing center. Cell 2006; 126:79-92.

9 Xie Z, Johansen LK, Gustafson AM, et al. Genetic and functional diversification of small RNA pathways in plants. PLoS Biol 2004; 2:E104.

10 Ye R, Wang W, Iki T, et al. Cytoplasmic assembly and selective nuclear import of Arabidopsis Argonaute4/siRNA complexes. Mol Cell 2012; 46:859-870.

11 Wierzbicki AT, Haag JR, Pikaard CS. Noncoding transcription by RNA polymerase Pol IVb/Pol V mediates transcriptional silencing of overlapping and adjacent genes. Cell 2008; 135:635-648

12 Wierzbicki AT, Ream TS, Haag JR, Pikaard CS. RNA polymerase $\mathrm{V}$ transcription guides ARGONAUTE4 to chromatin. Nat Genet 2009; 41:630-634.

13 Ream TS, Haag JR, Wierzbicki AT, et al. Subunit compositions of the RNA-silencing enzymes Pol IV and Pol V reveal their origins as specialized forms of RNA polymerase II. Mol Cell 2009; 33:192-203.

14 Huang L, Jones AM, Searle I, et al. An atypical RNA polymerase involved in RNA silencing shares small subunits with RNA polymerase II. Nat Struct Mol Biol 2009; 16:91-93.

15 Law JA, Ausin I, Johnson LM, et al. A protein complex required for polymerase $\mathrm{V}$ transcripts and RNA-directed DNA methylation in Arabidopsis. Curr Biol 2010; 20:951-956.

16 Zhong X, Hale CJ, Law JA, et al. DDR complex facilitates global association of RNA polymerase V to promoters and evolutionarily young transposons. Nat Struct Mol Biol 2012; 19:870-875.

17 Zhong X, Du J, Hale CJ, et al. Molecular mechanism of action of plant DRM de novo DNA methyltransferases. Cell 2014; 157:1050-1060.

18 Baumbusch LO, Thorstensen T, Krauss V, et al. The Arabidopsis thaliana genome contains at least 29 active genes encoding SET domain proteins that can be assigned to four evolutionarily conserved classes. Nucleic Acids Res 2001; 29:4319-4333.

19 Pontvianne F, Blevins T, Pikaard CS. Arabidopsis histone lysine methyltransferases. Adv Bot Res 2010; 53:1-22.

20 Rea S, Eisenhaber F, O'Carroll D, et al. Regulation of chromatin structure by site-specific histone $\mathrm{H} 3$ methyltransferases. Nature 2000; 406:593-599.

21 Jackson JP, Lindroth AM, Cao X, Jacobsen SE. Control of CpNpG DNA methylation by the KRYPTONITE histone H3 methyltransferase. Nature 2002; 416:556-560.

22 Ebbs ML, Bender J. Locus-specific control of DNA methylation by the Arabidopsis SUVH5 histone methyltransferase. Plant Cell 2006; 18:1166-1176.

23 Jackson JP, Johnson L, Jasencakova Z, et al. Dimethylation of histone $\mathrm{H} 3$ lysine 9 is a critical mark for DNA methylation and gene silencing in Arabidopsis thaliana. Chromosoma 2004; 112:308-315.

24 Johnson LM, Bostick M, Zhang X, et al. The SRA methyl-cytosine-binding domain links DNA and histone methylation. Curr Biol 2007; 17:379-384. 
25 Rajakumara E, Law JA, Simanshu DK, et al. A dual flip-out mechanism for $5 \mathrm{mC}$ recognition by the Arabidopsis SUVH5 SRA domain and its impact on DNA methylation and H3K9 dimethylation in vivo. Genes Dev 2011; 25:137-152.

26 Liu ZW, Shao CR, Zhang CJ, et al. The SET domain proteins SUVH2 and SUVH9 are required for Pol V occupancy at RNA-directed DNA methylation loci. PLoS Genet 2014; 10:e1003948.

27 Johnson LM, Du J, Hale CJ, et al. SRA- and SET-domain-containing proteins link RNA polymerase $\mathrm{V}$ occupancy to DNA methylation. Nature 2014; 507:124-128.

28 Thorstensen T, Fischer A, Sandvik SV, et al. The Arabidopsis SUVR4 protein is a nucleolar histone methyltransferase with preference for monomethylated H3K9. Nucleic Acids Res 2006; 34:5461-5470.

29 Veiseth SV, Rahman MA, Yap KL, et al. The SUVR4 histone lysine methyltransferase binds ubiquitin and converts H3K9me1 to H3K9me3 on transposon chromatin in Arabidopsis. PLoS Genet 2011; 7:e1001325.

30 Caro E, Stroud H, Greenberg MV, et al. The SET-domain protein SUVR5 mediates H3K9me2 deposition and silencing at stimulus response genes in a DNA methylation-independent manner. PLoS Genet 2012; 8:e1002995.

31 Jeddeloh JA, Stokes TL, Richards EJ. Maintenance of genomic methylation requires a SWI2/SNF2-like protein. Nature Genetics 1999; 22:94-97.

32 Kanno T, Mette MF, Kreil DP, Aufsatz W, Matzke M, Matzke AJ. Involvement of putative SNF2 chromatin remodeling protein DRD1 in RNA-directed DNA methylation. Curr Biol 2004; 14:801-805.

33 Smith LM, Pontes O, Searle I, et al. An SNF2 protein associated with nuclear RNA silencing and the spread of a silencing signal between cells in Arabidopsis. Plant Cell 2007; 19:1507-1521.

34 Zemach A, Kim MY, Hsieh PH, et al. The Arabidopsis nucleosome remodeler DDM1 allows DNA methyltransferases to access H1-containing heterochromatin. Cell 2013; 153:193205.

35 Smith LM, Baulcombe DC. Dissection of silencing signal movement in Arabidopsis. Plant Signal Behav 2007; 2:501502.

36 Law JA, Vashisht AA, Wohlschlegel JA, Jacobsen SE. SHH1, a homeodomain protein required for DNA methylation, as well as RDR2, RDM4, and chromatin remodeling factors, associate with RNA polymerase IV. PLoS Genet 2011; 7:e1002195.

37 Zhang H, Ma ZY, Zeng L, et al. DTF1 is a core component of RNA-directed DNA methylation and may assist in the recruitment of Pol IV. Proc Natl Acad Sci USA 2013; 110:82908295.

38 Amedeo P, Habu Y, Afsar K, Mittelsten Scheid O, Paszkowski J. Disruption of the plant gene MOM releases transcriptional silencing of methylated genes. Nature 2000; 405:203-206.

39 Yokthongwattana C, Bucher E, Caikovski M, et al. MOM1 and Pol-IV/V interactions regulate the intensity and specificity of transcriptional gene silencing. EMBO J 2010; 29:340351.

40 Numa H, Kim JM, Matsui A, et al. Transduction of RNA-directed DNA methylation signals to repressive histone marks in Arabidopsis thaliana. EMBO J 2010; 29:352-362.

41 Moissiard G, Cokus SJ, Cary J, et al. MORC family ATPases required for heterochromatin condensation and gene silencing. Science 2012; 336:1448-1451.

42 Lorkovic ZJ, Naumann U, Matzke AJ, Matzke M. Involvement of a GHKL ATPase in RNA-directed DNA methylation in Arabidopsis thaliana. Curr Biol 2012; 22:933-938.

43 Moissiard G, Bischof S, Husmann D, et al. Transcriptional gene silencing by Arabidopsis microrchidia homologues involves the formation of heteromers. Proc Natl Acad Sci USA 2014; 111:7474-7479.

44 Zhang CJ, Ning YQ, Zhang SW, et al. IDN2 and its paralogs form a complex required for RNA-directed DNA methylation. PLoS Genet 2012; 8:e1002693.

45 Xie M, Ren G, Costa-Nunes P, Pontes O, Yu B. A subgroup of SGS3-like proteins act redundantly in RNA-directed DNA methylation. Nucleic Acids Res 2012; 40:4422-4431.

46 Ausin I, Greenberg MV, Simanshu DK, et al. INVOLVED IN DE NOVO 2-containing complex involved in RNA-directed DNA methylation in Arabidopsis. Proc Natl Acad Sci USA 2012; 109:8374-8381.

47 Zhu Y, Rowley MJ, Bohmdorfer G, Wierzbicki AT. A SWI/ SNF chromatin-remodeling complex acts in noncoding RNA-mediated transcriptional silencing. Mol Cell 2013; 49:298-309.

48 Gong Z, Morales-Ruiz T, Ariza RR, Roldan-Arjona T, David L, Zhu JK. ROS1, a repressor of transcriptional gene silencing in Arabidopsis, encodes a DNA glycosylase/lyase. Cell 2002; 111:803-814.

49 He XJ, Hsu YF, Pontes O, et al. NRPD4, a protein related to the RPB4 subunit of RNA polymerase II, is a component of RNA polymerases IV and V and is required for RNA-directed DNA methylation. Genes Dev 2009; 23:318-330.

50 Liu Q, Gong Z. The coupling of epigenome replication with DNA replication. Curr Opin Plant Biol 2011; 14:187-194.

51 Liu J, Bai G, Zhang C, et al. An atypical component of RNA-directed DNA methylation machinery has both DNA methylation-dependent and -independent roles in locus-specific transcriptional gene silencing. Cell Res 2011; 21:16911700.

52 Zhu J, Kapoor A, Sridhar VV, Agius F, Zhu JK. The DNA glycosylase/lyase ROS1 functions in pruning DNA methylation patterns in Arabidopsis. Curr Biol 2007; 17:54-59.

53 Penterman J, Uzawa R, Fischer RL. Genetic interactions between DNA demethylation and methylation in Arabidopsis. Plant Physiol 2007; 145:1549-1557.

54 He XJ, Hsu YF, Zhu S, et al. An effector of RNA-directed DNA methylation in Arabidopsis is an ARGONAUTE 4- and RNA-binding protein. Cell 2009; 137:498-508.

55 Dou K, Huang CF, Ma ZY, et al. The PRP6-like splicing factor STA1 is involved in RNA-directed DNA methylation by facilitating the production of Pol V-dependent scaffold RNAs. Nucleic Acids Res 2013; 41:8489-8502.

56 He XJ, Hsu YF, Zhu S, et al. A conserved transcriptional regulator is required for RNA-directed DNA methylation and plant development. Genes Dev 2009; 23:2717-2722.

57 Huettel B, Kanno T, Daxinger L, Aufsatz W, Matzke AJ, Matzke M. Endogenous targets of RNA-directed DNA methylation and Pol IV in Arabidopsis. EMBO J 2006; 25:2828- 
2836.

58 Zilberman D, Cao X, Jacobsen SE. ARGONAUTE4 control of locus-specific siRNA accumulation and DNA and histone methylation. Science 2003; 299:716-719.

59 Henderson IR, Zhang X, Lu C, et al. Dissecting Arabidopsis thaliana DICER function in small RNA processing, gene silencing and DNA methylation patterning. Nat Genet 2006; 38:721-725.

60 Wang Y, Liu J, Xia R, et al. The protein kinase TOUSLED is required for maintenance of transcriptional gene silencing in Arabidopsis. EMBO Rep 2007; 8:77-83.

61 Shen J, Ren X, Cao R, Liu J, Gong Z. Transcriptional gene silencing mediated by a plastid inner envelope phosphoenolpyruvate/phosphate translocator CUE1 in Arabidopsis. Plant Physiol 2009; 150:1990-1996.

62 Charron JB, He H, Elling AA, Deng XW. Dynamic landscapes of four histone modifications during deetiolation in Arabidopsis. Plant Cell 2009; 21:3732-3748.

63 Roudier F, Ahmed I, Berard C, et al. Integrative epigenomic mapping defines four main chromatin states in Arabidopsis. EMBO J 2011; 30:1928-1938.

64 Trievel RC, Beach BM, Dirk LM, Houtz RL, Hurley JH. Structure and catalytic mechanism of a SET domain protein methyltransferase. Cell 2002; 111:91-103.

65 Blevins T, Pontvianne F, Cocklin R, et al. A two-step process for epigenetic inheritance in Arabidopsis. Mol Cell 2014; 54:30-42.
66 Chodavarapu RK, Feng S, Bernatavichute YV, et al. Relationship between nucleosome positioning and DNA methylation. Nature 2010; 466:388-392.

67 Cairns BR. The logic of chromatin architecture and remodelling at promoters. Nature 2009; 461:193-198.

68 Mosher RA, Schwach F, Studholme D, Baulcombe DC. PolIVb influences RNA-directed DNA methylation independently of its role in siRNA biogenesis. Proc Natl Acad Sci USA 2008; 105:3145-3150.

69 Law JA, Du J, Hale CJ, et al. Polymerase IV occupancy at RNA-directed DNA methylation sites requires SHH1. Nature 2013; 498:385-389.

70 Hetzl J, Foerster AM, Raidl G, Mittelsten Scheid O. CyMATE: a new tool for methylation analysis of plant genomic DNA after bisulphite sequencing. Plant J 2007; 51:526-536.

71 Zhang CJ, Zhou JX, Liu J, et al. The splicing machinery promotes RNA-directed DNA methylation and transcriptional silencing in Arabidopsis. EMBO J 2013; 32:1128-1140.

(Supplementary information is linked to the online version of the paper on the Cell Research website.)

(c) (i) This work is licensed under the CreativeCommons-Attribution 3.0 Unported License. To view a copy of this license, visit http://creativecommons.org/licenses/ by/3.0/legalcode 